\title{
Field theoretic renormalization study of reduced quantum electrodynamics and applications to the ultrarelativistic limit of Dirac liquids
}

\author{
S. Teber ${ }^{1}$ and A. V. Kotikov ${ }^{2}$ \\ ${ }^{1}$ Sorbonne Université, CNRS, Laboratoire de Physique Théorique et Hautes Energies, \\ LPTHE, F-75005 Paris, France \\ ${ }^{2}$ Bogoliubov Laboratory of Theoretical Physics, Joint Institute for Nuclear Research, \\ 141980 Dubna, Russia
}

(Received 5 February 2018; published 3 April 2018)

\begin{abstract}
The field theoretic renormalization study of reduced quantum electrodynamics (QED) is performed up to two loops. In the condensed matter context, reduced QED constitutes a very natural effective relativistic field theory describing (planar) Dirac liquids, e.g., graphene and graphenelike materials, the surface states of some topological insulators, and possibly half-filled fractional quantum Hall systems. From the field theory point of view, the model involves an effective (reduced) gauge field propagating with a fractional power of the d'Alembertian in marked contrast with usual QEDs. The use of the Bogoliubov-ParasiukHepp-Zimmermann prescription allows for a simple and clear understanding of the structure of the model. In particular, in relation with the ultrarelativistic limit of graphene, we straightforwardly recover the results for both the interaction correction to the optical conductivity $\mathcal{C}^{*}=\left(92-9 \pi^{2}\right) /(18 \pi)$ and the anomalous dimension of the fermion field $\gamma_{\psi}(\bar{\alpha}, \xi)=2 \bar{\alpha}(1-3 \xi) / 3-16\left(\zeta_{2} N_{F}+4 / 27\right) \bar{\alpha}^{2}+\mathrm{O}\left(\bar{\alpha}^{3}\right)$, where $\bar{\alpha}=$ $e^{2} /(4 \pi)^{2}$ and $\xi$ is the gauge-fixing parameter.
\end{abstract}

DOI: 10.1103/PhysRevD.97.074004

\section{INTRODUCTION}

It is well known that Dirac liquids possess an infrared (IR) Lorentz-invariant fixed point [1]. This fact is actually generic to systems with stable Fermi (or Dirac) points; see the textbook [2]. In these systems, low-energy excitations have a gapless linear, relativisticlike, spectrum as known theoretically for a long time in graphene $[3,4]$ and in the so-called spin-liquid phases of high temperature superconductors $[5,6]$. Such low-energy Dirac-like energy dispersions are by now well observed experimentally in various materials which are under active study, e.g., graphene [7], (artificial) graphenelike materials [8], surface states of topological insulators [9], the so-called DiracWeyl materials [10-15]; see the review [16] on these three-dimensional analogues of graphene and, very recently, half-filled fractional quantum Hall systems [17].

The IR Lorentz invariant fixed point arises from the longrange Coulomb interaction among the Dirac fermions which enforces the flow of the Fermi velocity, e.g., $v \approx$ $c / 300$ at experimentally accessible scales for graphene, to the velocity of light, $c$, in the IR with a corresponding flow

Published by the American Physical Society under the terms of the Creative Commons Attribution 4.0 International license. Further distribution of this work must maintain attribution to the author(s) and the published article's title, journal citation, and DOI. Funded by SCOAP . of the fine structure constant, e.g., $\alpha_{g} \approx e^{2} / 4 \pi \varepsilon \hbar v \approx 2.2$ for graphene, to the usual fine structure constant, $\alpha \approx 1 / 137$, in the IR. Moreover, in the case of planar systems such as graphene, the electrons are confined to a three-dimensional space-time, $d_{e}=2+1$, while interactions between them are mediated by four-dimensional photons, $d_{\gamma}=3+1$. The Lorentz invariant fixed point may therefore be effectively described by a massless relativistic quantum field theory $(\mathrm{QFT})$ model whereby $d_{e}$-dimensional fermions interact via a $d_{\gamma}$-dimensional $U(1)$ gauge field. Such a model belongs to the class of reduced quantum electrodynamics (QED) [18], reduced $\mathrm{QED}_{d_{y}, d_{e}}$ or simply $\mathrm{QED}_{d_{y}, d_{e}}$, also known as pseudo QED [19] and even more recently as mixed-dimensional QED [20]. Notice that in the particular case where gauge and fermion fields live in the same spacetime, $d_{\gamma}=d_{e}=d$, reduced QEDs correspond to the usual $\mathrm{QED}_{d}$ while in the reduced case $d_{e}<d_{\gamma}$. Early motivations for the study of reduced theories came from interest in branes [18,21], dynamical chiral symmetry breaking on a brane [18], conformal field theory [22] (and reference therein), as well as potential applications to condensed matter physics systems in relation with the quantum Hall effect and high temperature superconductivity $[19,23,24]$. In Ref. [25], $\mathrm{QED}_{d_{y}, d_{e}}$ was advocated as a minimal model to study the infrared Lorentz invariant fixed point of Dirac liquids with a special focus on $\mathrm{QED}_{4,3}$ relevant to intrinsic (or undoped) disorder-free graphene and similar planar 
materials. In the past years, there has been an increasing number of studies focusing on reduced QED and in particular $\mathrm{QED}_{4,3}$ in relation with, e.g., transport and spectral properties [26-29]; see also the short review [30], optical properties [31,32], quantum Hall effect [20,33,34], and dynamical chiral symmetry breaking $[35,36]$ in planar systems. Moreover, $\mathrm{QED}_{4,3}$ was shown to be unitary [37], its properties under the Landau-KhalatnikovFrandkin transformation were studied [38], its precise relation to $\mathrm{QED}_{3}$ was understood [35], it was shown to possess a strong-weak duality mapping the coupling constant $e$ to $\tilde{e}=8 \pi / e$ with a self-dual point at $e^{2}=8 \pi$ (or $\alpha=2$ ) [39], and, even more recently, it has been studied as an interacting boundary conformal field theory [40].

Though essentially of academic interest, a thorough understanding of the IR fixed point is a necessary prerequisite to set on a firm ground the study of the physics away from the fixed point which is closer to the experimental reality but more difficult to study theoretically. Indeed, in the nonrelativistic limit there is often no definitive agreement on the precise value of important quantities directly related to interaction effects; in relation with graphene, let us for example mention two quantities that have been the subject of extensive work during the past decade: the value of the interaction correction to the optical conductivity (see, e.g., Refs. [41-52]) and the value of the critical coupling constant for dynamical gap generation (see, e.g., Refs. [53-73]). It turns out that $\mathrm{QED}_{4,3}$ is an ideal playground to compute both of these quantities; see [25,26,28] (as well as [30] for a short review) and [35], respectively. The reason is that all the powerful multiloop machinery originally developed in particle physics and statistical mechanics to compute (massless) Feynman diagrams (see, e.g., Refs. [74,75] and also the lectures [76]) can be applied to reduced QED in order to rigorously understand the perturbative structure of the model as well as some of its nonperturbative features. Interestingly, the odd dimensionality of space-time together with the (related) presence of Feynman diagrams with noninteger indices brings a lot of novelties (as well as highly nontrivial additional complications) with respect to what is usually known from the study of $(3+1)$-dimensional theories; see Refs. [26,28,30,77] for a systematic computation of nontrivial master integrals in $\mathrm{QED}_{4,3}$ up to two loops. Besides loop calculations, a nontrivial aspect of reduced QED is related to the peculiar structure of its (sub)divergent graphs. It is then the purpose of renormalization to give a prescription on how to deal with these (sub)divergences. In all previous references, the so-called conventional renormalization has been used to achieve this purpose.

In this paper, we will focus on the field theoretic renormalization study of reduced quantum electrodynamics and in particular $\mathrm{QED}_{4,3}$. We will assume that all the needed master integrals are known and proceed in renormalizing the model and extracting anomalous dimensions and renormalized correlation functions with the help of the recursive subtraction scheme, the so-called $R$-operation, of Bogoliubov and Parasiuk [78] and Hepp [79] or its solution known as Zimmermann's forest formula [80] (see also the textbook Ref. [81] as well as [82]). The power of the Bogoliubov-Parasiuk-Hepp-Zimmermann (BPHZ) prescription, with respect to conventional renormalization, lies in the fact that it applies diagram by diagram, gives a very clear and unambiguous prescription on how to subtract (sub)divergences, and is conveniently automated $[85,86]$. We will apply such a prescription to computing the interaction correction to the optical conductivity and the anomalous dimension of the fermion field (which plays an important role with respect to the critical coupling constant for dynamical gap generation). As will be shown in detail in the following, the obtained results are in complete agreement with those obtained via conventional renormalization thereby lifting any possible ambiguity as to their value at the IR fixed point.

The paper is organized as follows. In Sec. II, we motivate the study of reduced QED and set up the general notations and conventions. In Sec. III, we recall the one-loop structure of the model. In Secs. IV and V, we then focus on the renormalization of the polarization operator and the fermion self-energy, respectively. We conclude in Sec. VI and define some basic master integrals appearing in the text in Appendix. In the following, we work in units where $\hbar=c=1$.

\section{GENERAL APPROACH AND MODEL}

\section{A. General approach}

The most general low-energy effective action (model I) describing a disorder-free intrinsic Dirac liquid reads (in Minkowski space)

$$
\begin{aligned}
S= & \int \mathrm{d} t \mathrm{~d}^{D_{e}} x\left[\bar{\psi}_{\sigma}\left(\mathrm{i} \gamma^{0} \partial_{t}+\mathrm{i} v \vec{\gamma} \cdot \vec{\nabla}\right) \psi^{\sigma}\right. \\
& \left.-e \bar{\psi}_{\sigma} \gamma^{0} A_{0} \psi^{\sigma}+e \frac{v}{c} \bar{\psi}_{\sigma} \vec{\gamma} \cdot \vec{A} \psi^{\sigma}\right] \\
& +\int \mathrm{d} t \mathrm{~d}^{D_{\gamma}} x\left[-\frac{1}{4} F^{\mu \nu} F_{\mu \nu}-\frac{1}{2 \xi}\left(\partial_{\mu} A^{\mu}\right)^{2}\right],
\end{aligned}
$$

where $\psi^{\sigma} \equiv \psi^{\sigma}(t, \vec{x})$ is a four component spinor field of spin index $\sigma$ which varies from 1 to $N_{F}\left(N_{F}=2\right.$ for graphene), $v$ is the Fermi velocity, $c$ is the velocity of light which is also implicitly contained in the gauge field action through $\partial_{\mu}=\left(\frac{1}{c} \partial_{t}, \vec{\nabla}\right), \xi$ is the gauge fixing parameter, and $\gamma^{\mu}$ is a $4 \times 4$ Dirac matrix satisfying the usual algebra: $\left\{\gamma^{\mu}, \gamma^{\nu}\right\}=2 g^{\mu \nu}$ where $g^{\mu \nu}=\operatorname{diag}(1,-1,-1, \ldots,-1)$ is the metric tensor in $D_{e}+1$ dimensions. The action (1) describes the coupling of a fermion field in $d_{e}=D_{e}+1$ dimensions with a $U(1)$ gauge field in $d_{\gamma}=D_{\gamma}+1$ dimensions. In the case of graphene, we have $D_{e}=2$ 
and $D_{\gamma}=3$, i.e., fermions in the plane and gauge field in the bulk. Because of the running of $v$ all the way up to $c$, any complete renormalization group analysis of Dirac materials should in principle be based on (1). It turns out that such a task is rather involved and, presently, very few results are available; see e.g., Refs. $[1,87]$.

In the literature, the overwhelming majority of works on Dirac liquids focuses on the nonrelativistic limit where $v / c \rightarrow 0$ (instantaneous interactions). This is, of course, a very realistic assumption given the smallness of the ratio, e.g., $v / c \approx 1 / 300$ for graphene, at the experimentally accessible scales. In this limit, there is no coupling to vector photons and Eq. (1) simplifies as (model II)

$$
\begin{aligned}
S= & \int \mathrm{d} t \mathrm{~d}^{D_{e}} x \bar{\psi}_{\sigma}\left[\gamma^{0}\left(\mathrm{i} \partial_{t}-e A_{0}\right)+\mathrm{i} v \vec{\gamma} \cdot \vec{\nabla}\right] \psi^{\sigma} \\
& +\frac{1}{2} \int \mathrm{d} t \mathrm{~d}^{D_{\gamma}} x\left(\vec{\nabla} A_{0}\right)^{2},
\end{aligned}
$$

where the Coulomb gauge is used. Most of the theoretical results derived on the basis of (2) are perturbative with expansions in the (bare) coupling constant reaching twoloop accuracy (some partial results are available at threeloop [88]). Of course, given the strength of the interaction in this limit, e.g., $\alpha_{g} \approx 2.2$ for graphene, such expansions may not be reliable and a nonperturbative treatment of the interactions seems to be required. Such treatments are in general limited to an random phase approximation-like resummation or leading order (LO) in the $1 / N$ expansion; see Ref. [89] for an attempt to compute next-to-leading order (NLO) corrections. Often, even LO results are approximate (using the so-called static approximation, neglecting Fermi velocity renormalization, etc.). So, despite the fact that (2) is simpler than (1), calculations are difficult to carry out in a rigorous way in this limit. This often results in a rather confusing situation where even the simplest quantities are subject to theoretical uncertainties as mentioned in the Introduction; see, e.g., Refs. [35,49] for examples and references therein.

In this paper, we will follow an alternative nonconventional route initiated in Refs. [25,26,28]. We will study interaction effects starting from the IR Lorentz invariant fixed point where $v / c \rightarrow 1$ and the interaction is fully retarded. In this limit, Eq. (1) can be written in covariant form as (model III)

$S=\int \mathrm{d}^{d_{e}} x \bar{\psi}_{\sigma} \mathrm{i} \not \supset \psi^{\sigma}+\int \mathrm{d}^{d_{\gamma}} x\left[-\frac{1}{4} F^{\mu \nu} F_{\mu \nu}-\frac{1}{2 \xi}\left(\partial_{\mu} A^{\mu}\right)^{2}\right]$,

where $D_{\mu}=\partial_{\mu}+\mathrm{i} e A_{\mu}$ is the covariant derivative. As anticipated in the Introduction, we will refer to this model as reduced QED [18] or $\mathrm{QED}_{d_{y}, d_{e}}$ for short. For $d_{e}=d_{\gamma}=d$, Eq. (3) simply reduces to the usual $\mathrm{QED}_{d}$. The peculiar case of $\mathrm{QED}_{4,3}$ describes graphene, and other planar Dirac liquids, at its Lorentz invariant fixed point. In this respect, model II corresponds to a nonrelativistic reduced $\mathrm{QED}_{d_{y}, d_{e}}\left(\mathrm{NRQED}_{d_{\gamma}, d_{e}}\right)$ while model III interpolates between I and II. From the field theoretic point of view, the model of Eq. (3) (and similarly for the two previous ones) is characterized by an effective free gaugefield action with fractional d'Alembertian [90,92]. The latter can be derived from Eq. (3) by integrating out the gauge degrees of freedom transverse to the $d_{e}$-dimensional manifold. Including fermions, the Lagrangian density $\mathcal{L}$ which is such that $S=\int \mathrm{d}^{d_{e}} x \mathcal{L}$, reads

$$
\begin{aligned}
\mathcal{L}= & \bar{\psi}_{\sigma} \mathrm{i}(\not \partial+\mathrm{i} e \tilde{A}) \psi^{\sigma}-\frac{1}{4} \tilde{F}^{\mu \nu} \frac{(4 \pi)^{\varepsilon_{e}}}{\Gamma\left(1-\varepsilon_{e}\right)[-\square]^{\varepsilon_{e}}} \tilde{F}_{\mu \nu} \\
& +\frac{1}{2 \tilde{\xi}} \tilde{A}^{\mu} \frac{(4 \pi)^{\varepsilon_{e}} \partial_{\mu} \partial_{\nu}}{\Gamma\left(1-\varepsilon_{e}\right)[-\square]^{\varepsilon_{e}}} \tilde{A}^{\nu},
\end{aligned}
$$

where we used the notation $\tilde{A}^{\mu}$ to emphasize the fact that it is a reduced gauge field (in $d_{e}$-dimensional space), $\varepsilon_{e}=\left(d_{\gamma}-d_{e}\right) / 2$ and $\tilde{\xi}=\varepsilon_{e}+\left(1-\varepsilon_{e}\right) \xi$; see Sec. II B for more on notations. Though a priori mainly of academic interest, the general motivation to consider reduced QED models is that relativistic invariance allows for a rigorous and systematic study of interaction effects as explained in the Introduction. We will therefore focus on a fieldtheoretic renormalization study of model III as a prerequisite to study model II and eventually model I [99].

\section{B. Model and conventions}

We now proceed with presenting the model and setting up our conventions and notations; see also Refs. [25,28]. The Feynman rules for model III, Eq. (3), are summarized in Fig. 1. The free massless fermion propagator and fermion-photon vertex are the standard ones,

$$
S_{0}(p)=\frac{\mathrm{i}}{\not p}, \quad \Gamma_{0}^{\mu}=\gamma^{\mu},
$$

and the reduced gauge field propagator reads [see also Eq. (4) where fractional powers appear explicitly at the level of the action]

$$
\tilde{D}_{0}^{\mu \nu}(q)=\frac{\mathrm{i}}{(4 \pi)^{\varepsilon_{e}}} \frac{\Gamma\left(1-\varepsilon_{e}\right)}{\left(-q^{2}\right)^{1-\varepsilon_{e}}}\left(g^{\mu \nu}-(1-\tilde{\xi}) \frac{q^{\mu} q^{\nu}}{q^{2}}\right),
$$

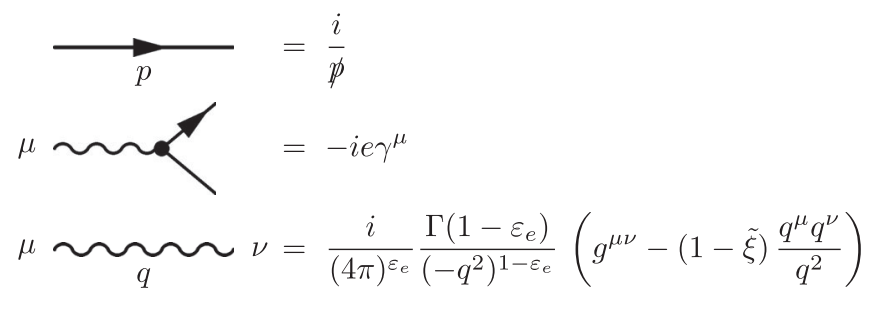

FIG. 1. Feynman rules for massless reduced $\mathrm{QED}_{d_{y}, d_{e}}$ (model III). 
where all components of momentum as well as the indices take their values in the $d_{e}$-dimensional space. The gauge fixing parameter of the reduced gauge field, $\tilde{\xi}=1-\tilde{\eta}$, is related to the gauge fixing parameter of the fourdimensional gauge field, $\xi=1-\eta$, with the help of

$$
\tilde{\xi}=\varepsilon_{e}+\left(1-\varepsilon_{e}\right) \xi, \quad \tilde{\eta}=\left(1-\varepsilon_{e}\right) \eta .
$$

The photon propagator, Eq. (6), can be separated in longitudinal and transverse parts which read

$$
\begin{aligned}
& \tilde{d}_{0 \|}\left(q^{2}\right)=\frac{\mathrm{i} \tilde{\xi}}{(4 \pi)^{\varepsilon_{e}}} \frac{\Gamma\left(1-\varepsilon_{e}\right)}{\left(-q^{2}\right)^{1-\varepsilon_{e}}}, \\
& \tilde{d}_{0 \perp}\left(q^{2}\right)=\frac{\mathrm{i}}{(4 \pi)^{\varepsilon_{e}}} \frac{\Gamma\left(1-\varepsilon_{e}\right)}{\left(-q^{2}\right)^{1-\varepsilon_{e}}} .
\end{aligned}
$$

In the case of $\mathrm{QED}_{4,3}, \varepsilon_{e}=1 / 2$ and the reduced propagator has a square root branch cut, whereas for $\mathrm{QED}_{4,2}, \varepsilon_{e}=1$ and the reduced propagator is logarithmic. Notice that the reduced $\mathrm{QED}_{4,2}$ models a one-dimensional system where fermions interact via the long-range (fully retarded) Coulomb interaction [18,22]. Another case is that of $\mathrm{QED}_{4,1}: \varepsilon_{e}=3 / 2$ which corresponds to a pointlike particle in a four-dimensional electromagnetic environment. In all cases the reduced QFT is nonlocal.

Switching on interactions, the dressed fermion propagator and fermion-photon vertex take the form

$$
\begin{gathered}
S(p)=\frac{\mathrm{i}}{\not p} \frac{1}{1-\Sigma_{V}\left(p^{2}\right)}, \\
\Gamma^{\mu}\left(p, p^{\prime}\right)=\gamma^{\mu}+\Lambda^{\mu}\left(p, p^{\prime}\right),
\end{gathered}
$$

where $\Sigma_{V}\left(p^{2}\right)$ is defined from the fermion self-energy,

$$
\Sigma(p)=\not p \Sigma_{V}\left(p^{2}\right),
$$

in a form appropriate to the massless case. As for the photon propagator, only its transverse part is affected by interactions as in the usual QED (with its precise form depending on $\varepsilon_{e}$ ),

$$
\begin{aligned}
& \tilde{d}_{\|}\left(q^{2}\right)=\tilde{d}_{0 \|}\left(q^{2}\right), \\
& \tilde{d}_{\perp}\left(q^{2}\right)=\tilde{d}_{0 \perp}\left(q^{2}\right) \frac{1}{1-\mathrm{i} q^{2} \tilde{d}_{0 \perp}\left(q^{2}\right) \Pi\left(q^{2}\right)},
\end{aligned}
$$

where $\Pi\left(q^{2}\right)$ is defined from the photon self-energy,

$$
\Pi^{\mu \nu}(q)=\left(g^{\mu \nu} q^{2}-q^{\mu} q^{\nu}\right) \Pi\left(q^{2}\right) .
$$

For arbitrary $d_{e}$ and $d_{\gamma}$ the dimensions of the fields and coupling are given by

$$
\begin{aligned}
& {[\psi]=\frac{d_{e}-1}{2}=\frac{3}{2}-\varepsilon_{e}-\varepsilon_{\gamma}, \quad\left[A^{\mu}\right]=\frac{d_{\gamma}-2}{2}=1-\varepsilon_{\gamma}} \\
& {[e]=2-\frac{d_{\gamma}}{2}=\varepsilon_{\gamma}}
\end{aligned}
$$

where the parameters $\varepsilon_{\gamma}$ and $\varepsilon_{e}$ read

$$
\varepsilon_{\gamma}=\frac{4-d_{\gamma}}{2}, \quad \varepsilon_{e}=\frac{d_{\gamma}-d_{e}}{2} .
$$

Alternatively, the dimensions can be expressed as

$$
d_{\gamma}=4-2 \varepsilon_{\gamma}, \quad d_{e}=4-2 \varepsilon_{e}-2 \varepsilon_{\gamma} .
$$

Notice that, in momentum space, we have

$$
\left[\tilde{A}^{\mu}(k)\right]=\frac{d_{e}}{2}-\left[A^{\mu}\right]=1-\varepsilon_{e},
$$

and $\varepsilon_{e}$ therefore appears as an anomalous dimension for the reduced gauge field in accordance with the form of the reduced gauge propagator; see Fig. 1. Accordingly, gauge fixing is nonlocal with the usual factor $1 / q^{4}$ appearing in the factor of $\xi$ replaced by $1 /\left(q^{2}\right)^{2-\varepsilon_{e}}$ for arbitrary $\varepsilon_{e}$. Hence, upon performing a gauge transformation, $A_{\mu}(x) \rightarrow$ $A_{\mu}(x)+\partial_{\mu} \varphi(x)$, the correlator of the $\varphi$-field, which is proportional to the longitudinal part of the photon Green's function, also becomes anomalous. Formally, this amounts to defining a nonlocal gauge transformation for the reduced gauge field,

$$
\tilde{A}_{\mu}(x) \rightarrow \tilde{A}_{\mu}(x)+\partial_{\mu}^{1+\varepsilon_{e}} \tilde{\varphi}(x),
$$

where $\partial_{\mu}^{1+\varepsilon_{e}}$ is a fractional derivative; see, e.g., Refs. [104,105].

Despite being nonlocal, all reduced models with a fourdimensional gauge field $\left(d_{\gamma}=4\right)$ are renormalizable as witnessed by the fact that the coupling constant is dimensionless in $\mathrm{QED}_{4, d_{e}}$ whatever space the fermion field lives in; see Eq. (13b). This is in agreement with the counting of ultraviolet (UV) divergences as the degree of divergence of a diagram $G, \omega(G)$, in $\mathrm{QED}_{d_{y}, d_{e}}$ does not depend on the number of vertices whatever value $d_{e}$ takes [25,28]. Moreover, the most superficially divergent amplitudes in $\mathrm{QED}_{4, d_{e}} \mathrm{~S}$ are the fermion self-energy and the fermiongauge vertex: $\omega\left(\Sigma_{V}\right)=0$ and $\omega(\Gamma)=0$, respectively; i.e., they are logarithmically divergent as in $\mathrm{QED}_{4}$. On the other hand, the degree of divergence of the photon self-energy is lowered in reduced QEDs; while it is logarithmic in $\mathrm{QED}_{4}$, $\omega(\Pi)=0$, it is convergent in $\mathrm{RQED}_{4,3}: \omega(\Pi)=-1$, where $\Pi \equiv \Pi\left(q^{2}\right)$; see Eq. (12). At this point, it is important to note that, according to Weinberg's theorem [106], a Feynman graph $G$ is absolutely convergent not only if its degree of divergence, $\omega(G)$, is negative but also if the 
degrees of divergence, $\omega(\gamma)$, associated with all of its subgraphs $\gamma$ are also negative. When considering multiloop diagrams, one often encounters diagrams with divergent subgraphs and dealing with these subdivergences is one of the central aspects of renormalization theory. This will be our focus in the following, with an extreme case in $\mathrm{QED}_{4,3}$ corresponding to an overall finite graph (the photon selfenergy) with divergent (fermion and fermion-gauge vertex) subgraphs.

We are now in a position to introduce the renormalization constants associated with a general model of $\mathrm{QED}_{4, d_{e}}$,

$$
\begin{aligned}
\psi & =Z_{\psi}^{1 / 2} \psi_{r}, \quad A=Z_{A}^{1 / 2} A_{r}, \\
e & =Z_{e} e_{r} \mu^{\varepsilon_{\gamma}}=\frac{Z_{\Gamma}}{Z_{\psi} Z_{A}^{1 / 2}} e_{r} \mu^{\varepsilon_{\gamma}}, \quad \xi=Z_{\xi} \xi_{r},
\end{aligned}
$$

where the subscript $r$ denotes renormalized quantities and the renormalization scale, $\mu$, has been introduced in such a way that $e_{r}$ is dimensionless in $d_{\gamma}=4-2 \varepsilon_{\gamma}$ dimensions. The latter is related to the corresponding parameter $\bar{\mu}$ in the modified minimal subtraction ( $\bar{M} S$ ) scheme with the help of

$$
\bar{\mu}^{2}=4 \pi e^{-\gamma_{E}} \mu^{2},
$$

where $\gamma_{E}$ is Euler's constant. The renormalization constants also relate renormalized and bare propagators as follows:

$$
\begin{gathered}
S(p ; \alpha, \xi)=Z_{\psi}\left(\alpha_{r}\right) S_{r}\left(p ; \alpha_{r}, \xi_{r}, \mu\right), \\
D^{\mu \nu}(q ; \alpha, \xi)=Z_{A}\left(\alpha_{r}\right) D_{r}^{\mu \nu}\left(q ; \alpha_{r}, \xi_{r}, \mu\right), \\
\Gamma^{\mu}\left(p, p^{\prime} ; \alpha, \xi\right)=Z_{\Gamma}^{-1}\left(\alpha_{r}\right) \Gamma_{r}^{\mu}\left(p, p^{\prime} ; \alpha_{r}, \xi_{r}, \mu\right),
\end{gathered}
$$

where the bare propagators do not depend on $\mu$. In the MS scheme, these constants take the simple form

$$
Z_{x}\left(\alpha_{r}, \xi_{r}\right)=1+\delta Z_{x}\left(\alpha_{r}, \xi_{r}\right)=1+\sum_{l=1}^{\infty} \sum_{j=1}^{l} Z_{x}^{(l, j)}\left(\xi_{r}\right) \frac{\alpha_{r}^{l}}{\varepsilon_{\gamma}^{j}},
$$

where $x \in\{\psi, A, e, \xi, \Gamma\}, \alpha_{r}=e_{r}^{2} /(4 \pi)$, and $l$ runs over the number of loops at which UV singularities are subtracted. In the MS scheme the $Z_{x}$ do not depend on momentum or mass; furthermore, the dependence on $\mu$ is only through $\alpha_{r}$ and/or $\xi_{r}$. So the $Z_{x}$ depend only on $\alpha_{r}(\mu), \varepsilon_{\gamma}$, and eventually $\xi_{r}(\mu)$.

From the renormalization constants, it is possible to compute the $\beta$-function,

$$
\beta\left(\alpha_{r}\right)=\left.\mu \frac{\partial \alpha_{r}}{\partial \mu}\right|_{B} \quad\left(Z_{\alpha}=Z_{e}^{2}\right),
$$

where the subscript $B$ indicates that bare parameters, which do not depend on $\mu$, are fixed. Explicitly, it reads

$\beta\left(\alpha_{r}\right)=-2 \varepsilon_{\gamma} \alpha_{r}+\sum_{l=0}^{\infty} \beta_{l} \alpha_{r}^{l+2}, \quad \beta_{l}=2(l+1) Z_{\alpha}^{(l+1,1)}$,

where the coefficients $\beta_{l}$ are completely determined by the simple $1 / \varepsilon_{\gamma}$ poles in $Z_{\alpha}$. Similarly, one may compute the field anomalous dimensions which are defined as

$\gamma_{x}\left(\alpha_{r}, \xi_{r}\right)=-\left.\mu \frac{\mathrm{d} \log Z_{x}\left(\alpha_{r}, \xi_{r}\right)}{\mathrm{d} \mu}\right|_{B} \quad(x \in\{\psi, A\})$.

The radiatively generated photon anomalous dimension is gauge invariant and reads

$\gamma_{A}\left(\alpha_{r}\right)=\sum_{l=0}^{\infty} \gamma_{A, l} \alpha_{r}^{l+1}, \quad \gamma_{A, l}=2(l+1) Z_{A}^{(l+1,1)}$.

In the case of the fermion anomalous dimension, we have

$$
\begin{aligned}
\gamma_{\psi}\left(\alpha_{r}, \xi_{r}\right) & =\sum_{l=0}^{\infty} \gamma_{\psi, l}\left(\xi_{r}\right) \alpha_{r}^{l+1}, \\
\gamma_{\psi, l}\left(\xi_{r}\right) & =2(l+1) Z_{\psi}^{(l+1,1)}\left(\xi_{r}\right) .
\end{aligned}
$$

As in usual QEDs, the renormalized constants are not all independent. The gauge noninvariant gauge-fixing term is not renormalized; hence, $Z_{\xi}=Z_{A}$. Moreover, the Ward identity,

$$
Z_{\psi}=Z_{\Gamma}
$$

holds [25] for arbitrary $d_{e}$ implying that $Z_{e}=Z_{A}^{-1 / 2}$. Finally, the free gauge-field action is nonlocal in the reduced case, and hence the gauge field is not renormalized [107], $Z_{A}=1$, which implies that $Z_{e}=1$. As a consequence, there is no radiatively generated photon anomalous dimension and the $\beta$-function is zero which implies that the coupling remains marginal to all orders in perturbation theory,

$$
\beta\left(\alpha_{r}\right)=0, \quad d_{e}<4,
$$

a fact reminiscent of the $(1+1)$-dimensional TomonagaLuttinger model $[108,109]$. Assuming that the coupling constant is weak enough that no dynamical mass is generated, the reduced QED of Refs. [18,35] is therefore conformally invariant.

As anticipated in the Introduction, in order to compute renormalization constants and renormalized correlators, we will use the BPHZ prescription [78-80] 


$$
\begin{aligned}
\mathcal{R} G & =(1-\mathcal{K}) \mathcal{R}^{\prime} G, \\
\mathcal{R}^{\prime} G & =G+\sum_{\bar{\Gamma}_{d} \neq \varnothing} \prod_{\gamma \in \bar{\Gamma}_{d}}\left(-\mathcal{K} \mathcal{R}^{\prime} \gamma\right) \star G / \bar{\Gamma}_{d},
\end{aligned}
$$

where $\mathcal{R} G$ corresponds to the finite (renormalized) graph $G$ with all divergences (both subdivergences and overall divergence) subtracted. In Eq. (29), $\mathcal{R}^{\prime}$ is the so-called incomplete $R$-operation because it subtracts only the subdivergences, $\bar{\Gamma}_{d}$ is the set of all subdivergent graphs which are disjoint (nested ones are not allowed), and the operator $\mathcal{K}$ is defined as

$$
\mathcal{K}\left(\sum_{n=-\infty}^{+\infty} \frac{c_{n}}{\varepsilon_{\gamma}^{n}}\right)=\sum_{n=1}^{+\infty} \frac{c_{n}}{\varepsilon_{\gamma}^{n}}
$$

Moreover, the notation $G / \bar{\Gamma}$ means that the subdiagrams contained in $G$ are shrunk to a vertex and the $\star$ operation amounts to substitute the counterterm in the integrand of the shrunk diagram. In the case of (at most) logarithmic graphs, the $\star$ operator reduces to simple multiplication and we may identify the counterterm with the renormalization constant, i.e., $Z(\gamma)=\mathcal{K} \mathcal{R}^{\prime} \gamma$.

\section{REDUCED QED AT ONE LOOP}

We now proceed with reviewing the one-loop structure of the model [25]. The one-loop fermion self-energy, polarization operators, and fermion-gauge field vertex (see Fig. 2) are defined as

$$
\begin{aligned}
\mathrm{i} \Pi_{1}^{\mu \nu}(q)= & -\int\left[\mathrm{d}^{d_{e}} k\right] \operatorname{Tr}\left[\left(-\mathrm{i} e \gamma^{\mu}\right) S_{0}(k)\left(-\mathrm{i} e \gamma^{\nu}\right)\right. \\
& \left.\times S_{0}(k+q)\right],
\end{aligned}
$$
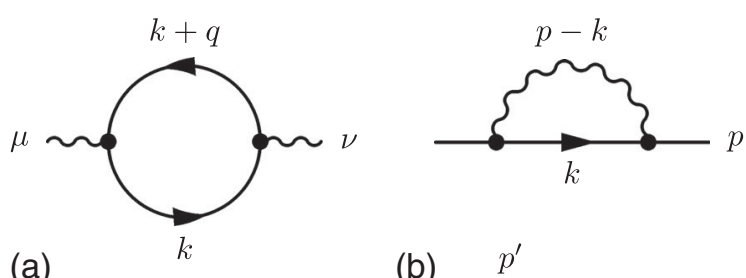

(a)

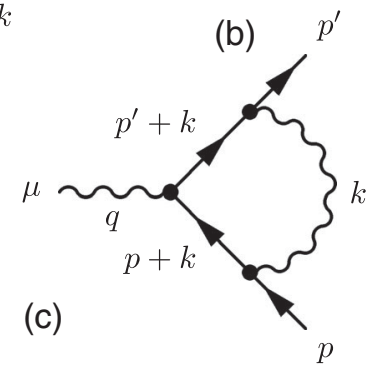

FIG. 2. One-loop diagrams: (a) gauge field self-energy, (b) fermion self-energy, and (c) fermion-gauge field vertex.

$$
\begin{aligned}
-\mathrm{i} \Sigma_{1}(p)= & \int\left[\mathrm{d}^{d_{e}} k\right]\left(-\mathrm{i} e \gamma^{\mu}\right) \tilde{D}_{0, \mu \nu}(p-k) S_{0}(k)\left(-\mathrm{i} e \gamma^{\nu}\right), \\
-\mathrm{i} e \Lambda_{1}^{\mu}\left(p, p^{\prime}\right)= & \int\left[\mathrm{d}^{d_{e}} k\right] \tilde{D}_{0}^{\alpha \beta}(p-k)\left(-\mathrm{i} e \gamma_{\alpha}\right) S_{0}(k)\left(-\mathrm{i} e \gamma^{\mu}\right) \\
& \times S_{0}(k+q)\left(-\mathrm{i} e \gamma_{\beta}\right) .
\end{aligned}
$$

The following parametrizations are useful:

$$
\begin{aligned}
\Pi\left(q^{2}\right) & =\frac{-\Pi_{\mu}^{\mu}(q)}{\left(d_{e}-1\right)\left(-q^{2}\right)}, \\
\Sigma_{V}\left(p^{2}\right) & =\frac{-1}{4 N_{F}\left(-p^{2}\right)} \operatorname{Tr}[\not p \Sigma(p)] .
\end{aligned}
$$

All calculations done (see Appendix for the master integrals), the results read

$$
\begin{aligned}
\Pi_{1}\left(q^{2} ; \bar{\alpha}\right)= & -4 N_{F} \bar{\alpha}\left(\frac{4 \pi}{-q^{2}}\right)^{\varepsilon_{e}}\left(\frac{\bar{\mu}^{2}}{-q^{2}}\right)^{\varepsilon_{\gamma}} \frac{d_{e}-2}{2\left(d_{e}-1\right)} \\
& \times e^{\gamma_{E} \varepsilon_{\gamma}} G\left(d_{e}, 1,1\right), \\
\Sigma_{1 V}\left(p^{2}\right)= & \bar{\alpha}\left(\frac{\bar{\mu}^{2}}{-p^{2}}\right)^{\varepsilon_{\gamma}} \Gamma\left(1-\varepsilon_{e}\right) \frac{d_{e}-2}{2}\left(\frac{\varepsilon_{e}}{d_{e}-2+\varepsilon_{e}}-\xi\right) \\
& \times e^{\gamma_{E} \varepsilon_{\gamma}} G\left(d_{e}, 1,1-\varepsilon_{e}\right), \\
\Lambda_{1}^{\mu}\left(p=p^{\prime}=0\right)= & \bar{\alpha} \gamma^{\mu}\left(\frac{\bar{\mu}^{2}}{m^{2}}\right)^{\varepsilon_{\gamma}}\left(\frac{\left(d_{e}-2\right)^{2}}{d_{e}\left(1-\varepsilon_{e}\right)}-(1-\xi)\right) \\
& \times e^{\gamma_{E} \varepsilon_{\gamma}} \frac{\Gamma\left(1+\varepsilon_{\gamma}\right)}{\varepsilon_{\gamma}},
\end{aligned}
$$

where $\bar{\alpha}=\alpha /(4 \pi)$, we have used the fact that $(1-\tilde{\xi})=$ $\left(1-\varepsilon_{e}\right)(1-\xi)$, and the vertex has been computed at $p=p^{\prime}=0$ with a small mass regulating an IR singularity. For later purposes, let us recall that the photon propagator (internal line in $\Sigma_{1}$ ) has a longitudinal and a transverse part; see Eq. (8). Then, Eq. (33b) shows that a similar decomposition holds for the one-loop fermion self-energy,

$$
\begin{aligned}
\Sigma_{1 V}\left(p^{2}\right)= & \Sigma_{1 V}^{(\|)}\left(p^{2}\right)+\Sigma_{1 V}^{(\perp)}\left(p^{2}\right), \\
\Sigma_{1 V}^{(\|)}\left(p^{2}\right)= & -\xi \bar{\alpha}\left(\frac{\bar{\mu}^{2}}{-p^{2}}\right)^{\varepsilon_{\gamma}} \Gamma\left(1-\varepsilon_{e}\right) \frac{d_{e}-2}{2} \\
& \times e^{\gamma_{E} \varepsilon_{\gamma}} G\left(d_{e}, 1,1-\varepsilon_{e}\right), \\
\Sigma_{1 V}^{(\perp)}\left(p^{2}\right)= & \varepsilon_{e} \bar{\alpha}\left(\frac{\bar{\mu}^{2}}{-p^{2}}\right)^{\varepsilon_{\gamma}} \Gamma\left(1-\varepsilon_{e}\right) \frac{d_{e}-2}{2\left(d_{e}-2+\varepsilon_{e}\right)} \\
& \times e^{\gamma_{E} \varepsilon_{\gamma}} G\left(d_{e}, 1,1-\varepsilon_{e}\right),
\end{aligned}
$$

where the transverse part is nonzero only in the reduced case. 
Focusing on $\mathrm{QED}_{4, d_{e}}$, the singular part of these selfenergies allows one to extract the one-loop counterterms which read

$$
\begin{aligned}
& \delta Z_{1 A}\left(\bar{\alpha}_{r}\right)=\mathcal{K}\left[\Pi_{1}\left(q^{2} ; \bar{\alpha}_{r}\right)\right] \\
& =\mathcal{K} \sim \mathcal{\bigcirc}]=0 \quad\left(d_{e}<4\right), \\
& \delta Z_{1 \psi}\left(\bar{\alpha}_{r}, \xi_{r}\right)=\mathcal{K}\left[\Sigma_{1 V}\left(p^{2} ; \bar{\alpha}_{r}, \xi_{r}\right)\right] \\
& =\mathcal{K}[\widetilde{\Omega}]=\bar{\alpha}_{r}\left(\frac{\varepsilon_{e}}{2-\varepsilon_{e}}-\xi_{r}\right) \frac{1}{\varepsilon_{\gamma}}, \\
& \delta Z_{1 \Gamma}\left(\bar{\alpha}_{r}, \xi_{r}\right)=-\mathcal{K}\left[\Lambda_{1}^{\mu}\left(p, p^{\prime} ; \bar{\alpha}_{r}, \xi_{r}\right) / \gamma^{\mu}\right] \\
& =-\mathcal{K}\left[\sim \varepsilon_{r} \sim \bar{\alpha}_{r}\left(\frac{\varepsilon_{e}}{2-\varepsilon_{e}}-\xi_{r}\right) \frac{1}{\varepsilon_{\gamma}}\right. \text {, }
\end{aligned}
$$

where the Lorentz structure of the graphs displayed in the brackets has been projected out. As anticipated in the last section, the Ward identity Eq. (27) is satisfied for all $d_{e}$. In graphical form, the latter reads at one-loop

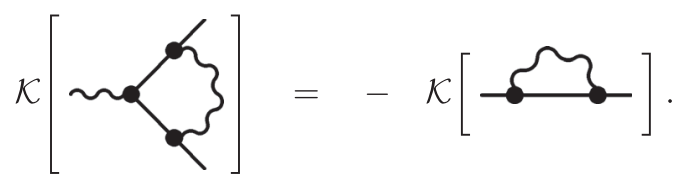

Explicitly, in the specific case of reduced $\mathrm{QED}_{4,3}$ $\left(\varepsilon_{e}=1 / 2\right.$ and $\left.\varepsilon_{\gamma} \rightarrow 0\right)$ the $\varepsilon_{\gamma}$-expansion of Eqs. (33a) and (33b) reads

$$
\begin{aligned}
\Pi_{1}\left(q^{2} ; \bar{\alpha}\right)=- & N_{F} \bar{\alpha} \frac{2 \pi^{2}}{\sqrt{-q^{2}}} \\
\times & {\left[1-\left(L_{q}-\log 4+1\right) \varepsilon_{\gamma}+\mathrm{O}\left(\varepsilon_{\gamma}^{2}\right)\right], } \\
\Sigma_{1 V}\left(p^{2} ; \bar{\alpha}, \xi\right)= & \bar{\alpha}\left[\frac{1-3 \xi}{3 \varepsilon_{\gamma}}-\frac{1-3 \xi}{3} \tilde{L}_{p}-2 \xi+\frac{10}{9}\right. \\
& +\left(\frac{1-3 \xi}{6}\left(\tilde{L}_{p}^{2}-7 \zeta_{2}\right)+2\left(\xi-\frac{5}{9}\right) \tilde{L}_{p}\right. \\
& \left.\left.-8 \xi+\frac{112}{27}\right) \varepsilon_{\gamma}+\mathrm{O}\left(\varepsilon_{\gamma}^{2}\right)\right],
\end{aligned}
$$

where $\tilde{L}_{x}=L_{x}+\log 4$. As a trivial application of the BPHZ prescription, by combining Eqs. (29) and (37), the renormalized one-loop self-energies read

$$
\begin{aligned}
\Pi_{1 r}\left(q^{2}\right) & =-\frac{N_{F} e^{2}}{8 \sqrt{-q^{2}}} \\
\Sigma_{1 V r}\left(p^{2}\right) & =-\bar{\alpha}_{r}\left(\frac{1-3 \xi_{r}}{3} \tilde{L}_{p}+2 \xi_{r}-\frac{10}{9}\right) .
\end{aligned}
$$

\section{TWO-LOOP POLARIZATION OPERATOR}

We now go on to two-loop order and first focus on the polarization operator. The total two-loop photon selfenergy can be decomposed as follows:

$$
\Pi_{2}^{\mu \nu}(q)=2 \Pi_{2 a}^{\mu \nu}(q)+\Pi_{2 b}^{\mu \nu}(q),
$$

where the diagrams are displayed in Fig. 3. The latter are defined as

$$
\begin{aligned}
\mathrm{i}_{2 a}^{\mu \nu}(q)= & -\int\left[\mathrm{d}^{d_{e}} k\right] \operatorname{Tr}\left[\left(-\mathrm{i} e \gamma^{\mu}\right) S_{0}(k+q)\left(-\mathrm{i} e \gamma^{\nu}\right)\right. \\
& \left.\times S_{0}(k)\left(-\mathrm{i} \not k \Sigma_{1 V}(k)\right) S_{0}(k)\right], \\
\mathrm{i}_{2 b}^{\mu \nu}(q)= & -\int\left[\mathrm{d}^{d_{e}} k\right] \operatorname{Tr}\left[\left(-\mathrm{i} e \gamma^{\nu}\right) S_{0}(k+q)\left(-\mathrm{i} e \Lambda_{1}^{\mu}(k, q)\right)\right. \\
& \left.\times S_{0}(k)\right],
\end{aligned}
$$

where the one-loop fermion self-energy and fermionphoton vertex were defined in Eq. (31). Because $\Pi^{\mu \nu}(q)$ is gauge independent, all calculations can be carried out in a specific gauge. In the following we shall work in the Feynman gauge, $\xi=1$.

All calculations done, the general expression for the twoloop photon self-energy diagrams of $\mathrm{QED}_{d_{\gamma}, d_{e}}$ read

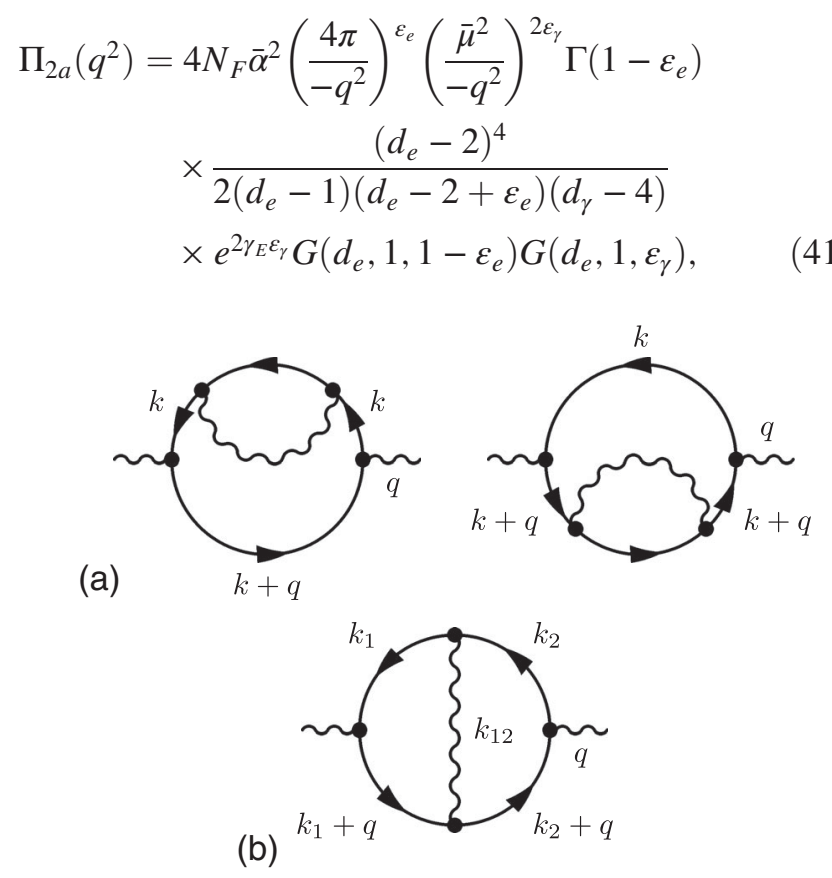

FIG. 3. Two-loop photon self-energy diagrams $\left(k_{12}=k_{1}-k_{2}\right)$. 


$$
\begin{aligned}
\Pi_{2 b}\left(q^{2}\right)= & -4 N_{F} \bar{\alpha}^{2}\left(\frac{4 \pi}{-q^{2}}\right)^{\varepsilon_{e}}\left(\frac{\bar{\mu}^{2}}{-q^{2}}\right)^{2 \varepsilon_{\gamma}} \Gamma\left(1-\varepsilon_{e}\right) \\
& \times \frac{d_{e}-2}{2\left(d_{e}-1\right)} e^{2 \gamma_{E} \varepsilon_{\gamma}}\left[2 G\left(d_{e}, 1,1-\varepsilon_{e}\right) G\left(d_{e}, 1, \varepsilon_{\gamma}\right) .\right. \\
& \times\left(d_{e}-4+\frac{2\left(d_{e}-2\right)^{3}}{d_{e}\left(d_{\gamma}-4\right)}-\frac{4\left(d_{e}-2\right)}{d_{e}+d_{\gamma}-6}-\frac{4\left(d_{e}-2\right)^{2}}{\left(d_{e}+d_{\gamma}-4\right)^{2}}\right. \\
& \left.+\frac{\left(d_{e}-2\right)\left(d_{e}^{2}-8\right)}{d_{e}\left(d_{e}+d_{\gamma}-4\right)}\right)-G\left(d_{e}, 1,1,1,1,1-\varepsilon_{e}\right) \\
& \left.\times\left(d_{e}-4+\frac{4\left(d_{e}-2\right)}{d_{e}+d_{\gamma}-6}-\frac{d_{e}\left(d_{e}-2\right)}{d_{e}+d_{\gamma}-4}\right)\right],
\end{aligned}
$$

where the two-loop master integral $G\left(d_{e}, 1,1,1,1, \alpha\right)$ with index $\alpha=1-\varepsilon_{e}$ appears; see Appendix. In order to compute the renormalized self-energies, we apply the BPHZ prescription Eq. (29). Graphically, the renormalization constants associated with each two-loop diagram read

$$
2 \delta Z_{2 a A}\left(\bar{\alpha}_{r}\right)=2 \mathcal{K}[\mathcal{K}[\mathcal{K}
$$

where, as in Eq. (35c), it is understood that the Lorentz structure of the diagrams in argument of $\mathcal{K}$ has been projected out. Because all graphs are at most logarithmic, the $\star$ operation reduces to a simple multiplication and will be omitted in the following. Upon computing the total renormalization constant, we see that the last terms in Eqs. (42a) and (42b) cancel each other thanks to the Ward identity (36). The total two-loop renormalization constant therefore reduces to

$$
\delta Z_{2 A}\left(\bar{\alpha}_{r}\right)=2 \mathcal{K}[\text { 2 }]+\mathcal{K}[\Omega \alpha],
$$

where $\delta Z_{2 A}=2 \delta Z_{2 a A}+\delta Z_{2 b A}$. Similar to the case of the usual QED [110], this simplification implies that, even though the individual diagrams have subdivergent graphs, the contributions of the latter cancel each other thanks to the Ward identity and their subtraction, which therefore does not affect the final result [111].
We now apply the above formulas to the case of $\mathrm{QED}_{4,3}$ $\left(\varepsilon_{e}=1 / 2\right.$ and $\left.\varepsilon_{\gamma} \rightarrow 0\right)$. From Eqs. (41a) and (41b), this leads to

$$
\begin{aligned}
& \Pi_{2 a}\left(q^{2}\right)=\frac{N_{F} \alpha^{2}}{\sqrt{-q^{2}}}\left[\frac{1}{12 \varepsilon_{\gamma}}-\frac{L_{q}}{6}+\frac{1}{9}+\mathrm{O}\left(\varepsilon_{\gamma}\right)\right], \\
& \Pi_{2 b}\left(q^{2}\right)=\frac{N_{F} \alpha^{2}}{\sqrt{-q^{2}}}\left[-\frac{1}{6 \varepsilon_{\gamma}}+\frac{L_{q}}{3}+\frac{\pi^{2}}{4}-\frac{25}{9}+\mathrm{O}\left(\varepsilon_{\gamma}\right)\right] .
\end{aligned}
$$

While the individual contributions are divergent (with only simple poles arising from divergent subgraphs; see below), the total two-loop polarization operator is finite $\left[\delta Z_{2 A}\left(\alpha_{r}\right)=0\right]$ and reads

$$
\Pi_{2}\left(q^{2}\right)=\Pi_{2 r}\left(q^{2}\right)=-N_{F} \frac{\alpha_{r}^{2}}{\sqrt{-q^{2}}} \frac{92-9 \pi^{2}}{36} .
$$

These results can also be recovered from the computation of individual counterterms in the Feynman gauge $(\xi=1)$,

$$
\begin{aligned}
\delta Z_{2 a A}\left(\alpha_{r}\right) & =\mathcal{K} \mathcal{R}^{\prime}\left[\Pi_{2 a}\left(q^{2}\right)\right] \\
& =\mathcal{K}\left[\Pi_{2 a}\left(q^{2}\right)\right]-\mathcal{K}\left[\mathcal{K}\left[\Sigma_{1 V}\left(\alpha_{r}\right)\right] \Pi_{1}\left(q^{2}\right)\right] \\
& =0 \\
\delta Z_{2 b A}\left(\alpha_{r}\right) & =\mathcal{K} \mathcal{R}^{\prime}\left[\Pi_{2 b}\left(q^{2} ; \alpha_{r}\right)\right] \\
& =\mathcal{K}\left[\Pi_{2 b}\left(q^{2} ; \alpha_{r}\right)\right]-2 \mathcal{K}\left[\mathcal{K}\left[\Lambda_{1}^{\mu}\left(\alpha_{r}\right) / \gamma^{\mu}\right] \Pi_{1}\left(q^{2} ; \alpha_{r}\right)\right] \\
& =0
\end{aligned}
$$

which vanish in accordance with the fact that the singularity of each two-loop photon self-energy graph in $\mathrm{QED}_{4,3}$ arises solely from its divergent subgraph. Hence, the renormalized diagrams read (for $\xi=1$ )

$$
\begin{aligned}
& \Pi_{2 a r}\left(q^{2} ; \alpha_{r}\right) \\
& =\Pi_{2 a}\left(q^{2} ; \alpha_{r}\right)-\mathcal{K}\left[\Sigma_{1 V}\left(\alpha_{r}\right)\right] \Pi_{1}\left(q^{2} ; \alpha_{r}\right)-\underbrace{\delta Z_{2 a A}\left(\alpha_{r}\right)}_{=0} \\
& =\frac{\alpha^{2}}{\sqrt{-q^{2}}}\left(-\frac{\tilde{L}_{q}}{12}+\frac{1}{9}+\frac{1}{12}\right), \\
& \Pi_{2 b r}\left(q^{2} ; \alpha_{r}\right) \\
& =\Pi_{2 b}\left(q^{2} ; \alpha_{r}\right)-2 \mathcal{K}\left[\Lambda_{1}^{\mu}\left(\alpha_{r}\right) / \gamma^{\mu}\right] \Pi_{1}\left(q^{2} ; \alpha_{r}\right)-\underbrace{\delta Z_{2 b A}\left(\alpha_{r}\right)}_{=0} \\
& =\frac{\alpha^{2}}{\sqrt{-q^{2}}}\left(\frac{\tilde{L}_{q}}{6}+\frac{\pi^{2}}{4}-\frac{25}{9}-\frac{1}{6}\right),
\end{aligned}
$$

where again $\tilde{L}_{q}=L_{q}+\log (4)$. Upon taking the sum of the individual contributions, Eq. (45) is straightforwardly recovered. 


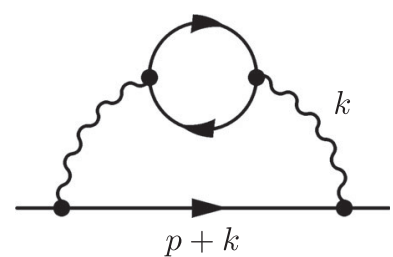

(a)

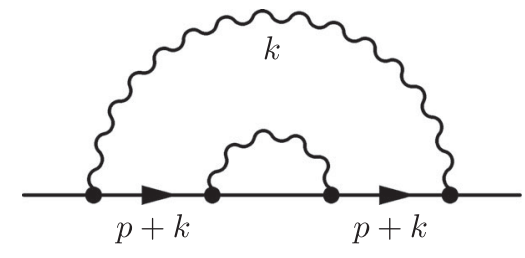

(b)

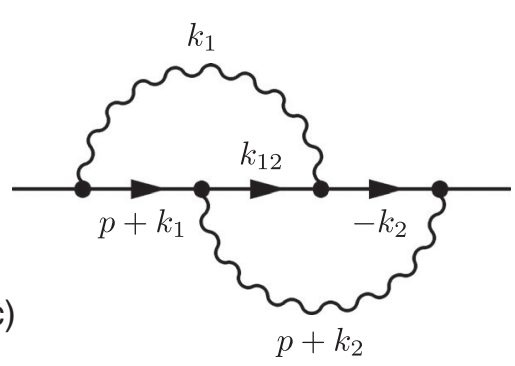

FIG. 4. Two-loop fermion self-energy diagrams $\left(k_{12}=k_{1}-k_{2}\right)$.

Finally, from the one-loop (38) and two-loop (45) results, the total renormalized polarization operator up to two loops can be written as

$\Pi_{r}\left(q^{2}\right)=\Pi_{1 r}\left(q^{2}\right)\left(1+\alpha_{r} \mathcal{C}^{*}+\mathrm{O}\left(\alpha_{r}^{2}\right)\right), \quad \mathcal{C}^{*}=\frac{92-9 \pi^{2}}{18 \pi}$,

where we recover the interaction correction coefficient $\mathcal{C}^{*}$ [25,26,30,113]. Combining Eqs. (48) and (8b), the transverse photon propagator up to two loops then reads

$$
d_{r \perp}\left(q^{2}\right)=\frac{\mathrm{i}}{2 \sqrt{-q^{2}}} \frac{1}{1+N_{F} \frac{\alpha_{r} \pi}{4}\left(1+\alpha_{r} \mathcal{C}^{*}\right)},
$$

and essentially remains free.

\section{TWO-LOOP FERMION SELF-ENERGY}

We may proceed in a similar way for the two-loop fermion self-energy,

$$
\Sigma_{2}(p)=\Sigma_{2 a}(p)+\Sigma_{2 b}(p)+\Sigma_{2 c}(p)
$$

where the diagrams are represented in Fig. 4. The latter are defined as

$$
\begin{aligned}
-\mathrm{i} \Sigma_{2 a}(p)= & \int\left[\mathrm{d}^{d_{e}} k\right]\left(-\mathrm{i} e \gamma^{\alpha}\right) S_{0}(p+k)\left(-\mathrm{i} e \gamma^{\beta}\right) \\
& \times \tilde{D}_{0 \alpha \mu}(k)\left(\mathrm{i} \Pi_{1}^{\mu \nu}(k)\right) \tilde{D}_{0 \nu \beta}(k), \\
-\mathrm{i} \Sigma_{2 b}(p)= & \int\left[\mathrm{d}^{d_{e}} k\right]\left(-\mathrm{i} e \gamma^{\mu}\right) S_{0}(p+k)\left(-\mathrm{i} \Sigma_{1}(p+k)\right) \\
& \times S_{0}(p+k)\left(-\mathrm{i} e \gamma^{\nu}\right) \tilde{D}_{0 \mu \nu}(k), \\
-\mathrm{i} \Sigma_{2 c}(p)= & \int\left[\mathrm{d}^{d_{e}} k\right]\left(-\mathrm{i} e \gamma^{\mu}\right) S_{0}(k)\left(-\mathrm{i} e \Lambda_{1}^{\mu}(k, p)\right) \\
& \times \tilde{D}_{0 \beta \mu}(k+p),
\end{aligned}
$$

with the one-loop polarization operator, fermion selfenergy, and fermion-photon vertex defined in Eq. (31).

All calculations done, the general expression for the twoloop photon self-energy diagrams of $\mathrm{QED}_{d_{y}, d_{e}}$ read

$$
\begin{aligned}
\Sigma_{V 2 a}\left(p^{2}\right)= & 4 N_{F} \bar{\alpha}^{2}\left(\frac{\bar{\mu}^{2}}{-p^{2}}\right)^{2 \varepsilon_{\gamma}} \Gamma^{2}\left(1-\varepsilon_{e}\right) \frac{\left(d_{e}-2\right)^{2}}{2\left(2 d_{\gamma}-d_{e}-6\right)} e^{2 \gamma_{E} \varepsilon_{\gamma}} G\left(d_{e}, 1,1\right) G\left(d_{e}, 1, \varepsilon_{\gamma}-\varepsilon_{e}\right), \\
\Sigma_{V 2 b}\left(p^{2}\right)= & \bar{\alpha}^{2}\left(\frac{\bar{\mu}^{2}}{-p^{2}}\right)^{2 \varepsilon_{\gamma}} \Gamma^{2}\left(1-\varepsilon_{e}\right) \frac{\left(d_{e}-2\right)\left(d_{\gamma}-3\right)\left(d_{\gamma}+d_{e}-4\right)}{2\left(d_{\gamma}-4\right)}\left(\xi-\frac{d_{\gamma}-d_{e}}{d_{\gamma}+d_{e}-4}\right)^{2} \\
& \times e^{2 \gamma_{E} \varepsilon_{\gamma}} G\left(d_{e}, 1,1-\varepsilon_{e}\right) G\left(d_{e}, 1-\varepsilon_{e}, \varepsilon_{\gamma}\right), \\
\Sigma_{V 2 c}\left(p^{2}\right)= & -\bar{\alpha}^{2}\left(\frac{\bar{\mu}^{2}}{-p^{2}}\right)^{2 \varepsilon_{\gamma}} \Gamma^{2}\left(1-\varepsilon_{e}\right) \frac{d_{e}-2}{2} e^{2 \gamma_{E} \varepsilon_{\gamma}} \\
& \times\left\{\left[d_{e}-4+\frac{\left(d_{e}-2\right)\left(d_{\gamma}-3 d_{e}+4\right)}{2\left(d_{\gamma}+d_{e}-4\right)}-\frac{\left(d_{\gamma}+d_{e}-6\right)\left(d_{\gamma}\left(d_{e}-4\right)+8\right)}{\left(2 d_{\gamma}+d_{e}-10\right)\left(2 d_{\gamma}+d_{e}-8\right)}\right.\right. \\
& -\frac{4\left(d_{\gamma}-d_{e}\right)}{d_{\gamma}+d_{e}-4}-\frac{d_{\gamma}-d_{e}}{2 d_{\gamma}+d_{e}-8}\left(d_{e}-8-4 \frac{d_{\gamma}+d_{e}-6}{d_{\gamma}+d_{e}-4}\right) \\
& \left.-\xi \frac{\left(d_{e}-2\right)\left(d_{\gamma}-d_{e}\right)}{d_{\gamma}+d_{e}-4}+\xi^{2} \frac{d_{e}-2}{2}\right] G^{2}\left(d_{e}, 1,1-\varepsilon_{e}\right)
\end{aligned}
$$




$$
\begin{aligned}
& +\left[2 d_{e}-d_{\gamma}-1+\frac{4\left(d_{e}-2\right)\left(d_{\gamma}-1\right)}{d_{\gamma}+d_{e}-4}+\frac{8\left(d_{\gamma}-1\right)}{d_{\gamma}-4}+\frac{2\left(d_{e}-8\right)\left(d_{\gamma}-d_{e}\right)}{d_{\gamma}+d_{e}-6}-\frac{4\left(d_{\gamma}-2\right)\left(d_{\gamma}-d_{e}\right)}{\left(d_{\gamma}-4\right)\left(d_{\gamma}+d_{e}-4\right)}\right. \\
& \left.+2 \xi \frac{\left(d_{\gamma}-3\right)\left(d_{\gamma}-d_{e}\right)}{d_{\gamma}+d_{e}-4}-\xi^{2}\left(d_{\gamma}-3\right)\right] G\left(d_{e}, 1,1-\varepsilon_{e}\right) G\left(1-\varepsilon_{e}, \varepsilon_{\gamma}\right) \\
& \left.-\frac{\left(d_{\gamma}-4\right)\left(d_{\gamma}\left(d_{e}-4\right)+8\right)}{\left(2 d_{\gamma}+d_{e}-8\right)\left(2 d_{\gamma}+d_{e}-10\right)} G\left(d_{e}, 1-\varepsilon_{e}, 1,1-\varepsilon_{e}, 1,1\right)\right\},
\end{aligned}
$$

where, in the last term, the complicated (UV convergent) diagram (see Appendix) comes with a factor $d_{\gamma}-4$ and will therefore not contribute in the case of $\mathrm{QED}_{4, d_{e}}$.

On the basis of these results, the computation of the renormalized fermion self-energies can be conveniently carried out using the BPHZ prescription Eq. (29). Graphically, the renormalization constants associated with the individual two-loop diagrams read

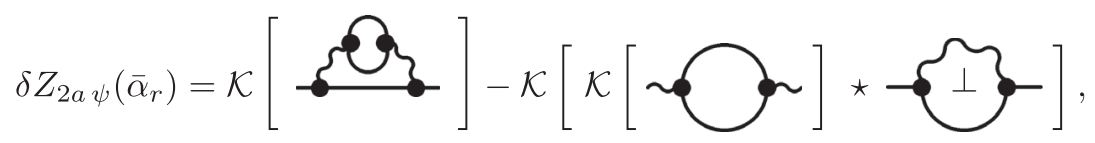

$$
\begin{aligned}
& \left.\delta Z_{2 b \psi}\left(\bar{\alpha}_{r}, \xi_{r}\right)=\mathcal{K}[\widetilde{\Omega}\}\right]-\mathcal{K}[\mathcal{K}[\widetilde{q}] * \widetilde{r}],
\end{aligned}
$$

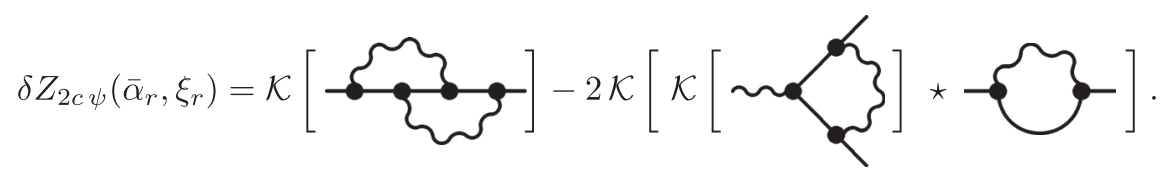

Notice that in Eq. (53a) the contraction of the one-loop polarization operator subgraph (which is transverse due to current conservation) resulted in the appearance of the transverse part of the one-loop fermion self-energy Eq. (34c). This is an example well known in the literature (see, e.g., Ref. [117]) of the sensitivity of the contraction procedure to the Lorentz structure of subdiagrams. Interestingly, the transverse part is nonzero only in the reduced case $\left(\varepsilon_{e}>0\right)$. However, in this case the photon self-energy is finite. It therefore vanishes in $\mathrm{QED}_{4, d_{e}}$ for all values of $d_{e}$. Taking this into account and summing all individual contributions, the total two-loop fermion renormalization constant reads

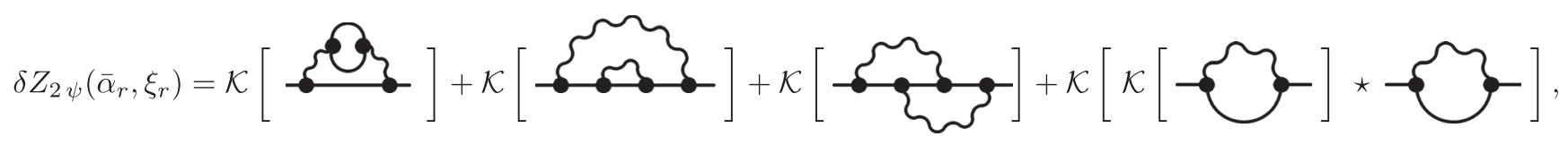

where we have used the Ward identity Eq. (36). In contrast to the case of the polarization operator, Eq. (54) shows that subdivergent graphs do contribute to the renormalization of the fermion self-energy (via the last term).

We now apply the above formulas to specific cases of $\mathrm{QED}_{4,3}\left(\varepsilon_{e}=1 / 2\right.$ and $\left.\varepsilon_{\gamma} \rightarrow 0\right)$. From Eqs. (52a), (52b), and (52c), this leads to

$$
\begin{aligned}
\Sigma_{2 a V}\left(p^{2} ; \bar{\alpha}, \xi\right)= & -\frac{2 \pi^{2} N_{F} \bar{\alpha}^{2}}{3}\left[\frac{1}{\varepsilon_{\gamma}}-2 L_{p}+\mathrm{O}\left(\varepsilon_{\gamma}\right)\right], \\
\Sigma_{2 b V}\left(p^{2} ; \bar{\alpha}, \xi\right)= & \bar{\alpha}^{2}\left[\frac{(1-3 \xi)^{2}}{18 \varepsilon_{\gamma}^{2}}+\frac{1-3 \xi}{\varepsilon_{\gamma}}\left(\frac{11}{27}-\frac{7 \xi}{9}-\frac{1-3 \xi}{9} \tilde{L}_{p}\right)+\frac{(1-3 \xi)^{2}}{9}\left(\tilde{L}_{p}^{2}-\frac{9}{2} \zeta_{2}\right)\right. \\
& \left.-\frac{2(11+9 \xi(7 \xi-6))}{27} \tilde{L}_{p}+\frac{2(103+81 \xi(7 \xi-6))}{81}+\mathrm{O}\left(\varepsilon_{\gamma}\right)\right],
\end{aligned}
$$




$$
\begin{aligned}
\Sigma_{2 c V}\left(p^{2} ; \bar{\alpha}, \xi\right)= & \bar{\alpha}^{2}\left[-\frac{(1-3 \xi)^{2}}{9 \varepsilon_{\gamma}^{2}}+\frac{1}{\varepsilon_{\gamma}}\left(\frac{2(1-3 \xi)^{2}}{9} \tilde{L}_{p}+\frac{(34-39 \xi) \xi}{9}-\frac{37}{27}\right)-\frac{2(1-3 \xi)^{2}}{9} \tilde{L}_{p}^{2}\right. \\
& \left.+\frac{2(37+3 \xi(39 \xi-34))}{27} \tilde{L}_{p}+\frac{71+21 \xi(3 \xi-2)}{9} \zeta_{2}-\frac{2\left(695-798 \xi+891 \xi^{2}\right)}{81}+\mathrm{O}\left(\varepsilon_{\gamma}\right)\right]
\end{aligned}
$$

which are valid for an arbitrary gauge fixing parameter $\xi$. Combining Eq. (55) with Eq. (53), the individual counterterms read

$$
\begin{aligned}
\delta Z_{2 a \psi}\left(\bar{\alpha}_{r}\right) & =\mathcal{K}\left[\Sigma_{2 a V}\left(p^{2} ; \bar{\alpha}_{r}\right)\right]-\mathcal{K}[\underbrace{\mathcal{K}\left[\Pi_{1}\left(q^{2} ; \bar{\alpha}_{r}, \xi_{r}\right)\right]}_{=0} \Sigma_{1 V}^{(\perp)}\left(p^{2} ; \bar{\alpha}_{r}, \xi_{r}\right)] \\
& =-\frac{2 \pi^{2} N_{F} \bar{\alpha}_{r}^{2}}{3 \varepsilon_{\gamma}}, \\
\delta Z_{2 b \psi}\left(\bar{\alpha}_{r}, \xi_{r}\right) & =\mathcal{K}\left[\Sigma_{2 b V}\left(p^{2} ; \bar{\alpha}_{r}, \xi_{r}\right)\right]-\mathcal{K}\left[\mathcal{K}\left(\Sigma_{1 V}\left(p^{2} ; \bar{\alpha}_{r}, \xi_{r}\right)\right) \Sigma_{1 V}\left(p^{2} ; \bar{\alpha}_{r}, \xi_{r}\right)\right] \\
& =-\frac{\left(1-3 \xi_{r}\right)^{2} \bar{\alpha}_{r}^{2}}{18}\left(\frac{1}{\varepsilon_{\gamma}^{2}}-\frac{2}{3 \varepsilon_{\gamma}}\right), \\
\delta Z_{2 c \psi}\left(\bar{\alpha}_{r}, \xi_{r}\right) & =\mathcal{K}\left[\Sigma_{2 c V}\left(p^{2} ; \bar{\alpha}_{r}, \xi_{r}\right)\right]+2 \mathcal{K}\left[\mathcal{K}\left(\Sigma_{1 V}\left(p^{2} ; \bar{\alpha}_{r}, \xi_{r}\right)\right) \Sigma_{1 V}\left(p^{2} ; \bar{\alpha}_{r}, \xi_{r}\right)\right] \\
& =\bar{\alpha}_{r}^{2}\left(\frac{\left(1-3 \xi_{r}\right)^{2}}{9 \varepsilon_{\gamma}^{2}}-\frac{17-6 \xi_{r}+9 \xi_{r}^{2}}{27 \varepsilon_{\gamma}}\right),
\end{aligned}
$$

where, in the first line, we have used the fact that $\Pi_{1}\left(q^{2}\right)$ is finite in $\mathrm{QED}_{4,3}$. The sum of Eq. (56) yields the total counterterm at two-loop order,

$$
\delta Z_{2 \psi}=-\frac{4 \bar{\alpha}_{r}^{2}}{\varepsilon_{\gamma}}\left(\zeta_{2} N_{F}+\frac{4}{27}\right)+\frac{\left(1-3 \xi_{r}\right)^{2} \bar{\alpha}_{r}^{2}}{18 \varepsilon^{2}}
$$

The individual renormalized diagrams are also straightforward to compute and read

$$
\begin{aligned}
\Sigma_{2 a V r}\left(p^{2} ; \bar{\alpha}_{r}\right) & =\Sigma_{2 a V}\left(p^{2} ; \bar{\alpha}_{r}\right)-\underbrace{\mathcal{K}\left[\Pi_{1}\left(q^{2} ; \bar{\alpha}_{r}, \xi_{r}\right)\right]}_{=0} \Sigma_{1 V}^{(\perp)}\left(p^{2} ; \bar{\alpha}_{r}, \xi_{r}\right)-\delta Z_{2 a \psi}\left(\bar{\alpha}_{r}\right) \\
& =8 N_{F} \zeta_{2} \bar{\alpha}_{r}^{2} L_{p}, \\
\Sigma_{2 b V r}\left(p^{2} ; \bar{\alpha}_{r}, \xi_{r}\right) & =\Sigma_{2 b V}\left(p^{2} ; \bar{\alpha}_{r}, \xi_{r}\right)-\mathcal{K}\left[\Sigma_{1 V}\left(p^{2} ; \bar{\alpha}_{r}, \xi_{r}\right)\right] \Sigma_{1 V}\left(p^{2} ; \bar{\alpha}_{r}, \xi_{r}\right)-\delta Z_{2 b \psi}\left(\bar{\alpha}_{r}, \xi_{r}\right) \\
& =\bar{\alpha}^{2}\left[\frac{\left(1-3 \xi_{r}\right)^{2}}{18}\left(\tilde{L}_{p}^{2}-2 \zeta_{2}\right)-\frac{4\left(1+\xi_{r}\left(6 \xi_{r}-5\right)\right)}{9} \tilde{L}_{p}+\frac{2\left(47-210 \xi_{r}+243 \xi_{r}^{2}\right)}{81}\right], \\
\Sigma_{2 c V r}\left(p^{2} ; \bar{\alpha}_{r}, \xi_{r}\right) & =\Sigma_{2 c V r}\left(p^{2} ; \bar{\alpha}_{r}, \xi_{r}\right)+2 \mathcal{K}\left[\Sigma_{1 V}\left(p^{2} ; \bar{\alpha}_{r}, \xi_{r}\right)\right] \Sigma_{1 V}\left(p^{2} ; \bar{\alpha}_{r}, \xi_{r}\right)-\delta Z_{2 c \psi}\left(\bar{\alpha}_{r}, \xi_{r}\right) \\
& =\bar{\alpha}^{2}\left[-\frac{\left(1-3 \xi_{r}\right)^{2}}{9} \tilde{L}_{p}^{2}+\frac{64}{9} \zeta_{2}+\frac{2\left(3+\xi_{r}\left(7 \xi_{r}-6\right)\right)}{3} \tilde{L}_{p}+\frac{2 \xi_{r}\left(82-81 \xi_{r}\right)}{27}-\frac{1166}{81}\right],
\end{aligned}
$$

where $\tilde{L}_{p}=L_{p}+\log 4$. The sum of Eq. (58) yields the total two-loop renormalized fermion self-energy,

$$
\Sigma_{2 V r}\left(p^{2} ; \bar{\alpha}_{r}, \xi_{r}\right)=\bar{\alpha}_{r}^{2}\left[8 N_{F} \zeta_{2} L_{p}-\frac{\left(1-3 \xi_{r}\right)^{2}}{18} \tilde{L}_{p}^{2}+\frac{64-\left(1-3 \xi_{r}\right)^{2}}{9} \zeta_{2}+\frac{2\left(7+\xi_{r}\left(9 \xi_{r}-8\right)\right)}{9} \tilde{L}_{p}-\frac{8\left(134-9 \xi_{r}\right)}{81}\right] .
$$

Combining the above two-loop results with the one-loop ones derived in the previous paragraphs, we recover the expression of the anomalous dimension of the fermion field up to two loops [28,118], 
$\gamma_{\psi}\left(\bar{\alpha}_{r}, \xi_{r}\right)=2 \bar{\alpha}_{r} \frac{1-3 \xi_{r}}{3}-16\left(\zeta_{2} N_{F}+\frac{4}{27}\right) \bar{\alpha}_{r}^{2}+\mathrm{O}\left(\bar{\alpha}_{r}^{3}\right)$,

where the two-loop contribution is gauge invariant. Combining (60) and (9a), the expansion of the renormalized fermion propagator up to two loops reads

$$
\begin{aligned}
-\mathrm{i} \not p S_{r}(p)= & 1+\bar{\alpha}_{r}\left(\frac{10}{9}-2 \xi_{r}-\frac{1-3 \xi_{r}}{3} \tilde{L}_{p}\right) \\
& +\bar{\alpha}_{r}^{2}\left(8 N_{F} \zeta_{2} L_{p}+\frac{\left(1-3 \xi_{r}\right)^{2}}{18} \tilde{L}_{p}^{2}\right. \\
& +\frac{2\left(11+3 \xi_{r}\left(8-9 \xi_{r}\right)\right)}{27} \tilde{L}_{p} \\
& -\frac{4\left(27+\xi_{r}\left(8-9 \xi_{r}\right)\right)}{9} \\
& \left.+\zeta_{2}\left(7+\frac{2 \xi_{r}}{3}-\xi_{r}^{2}\right)\right)+\mathrm{O}\left(\bar{\alpha}_{r}^{3}\right) .
\end{aligned}
$$

\section{CONCLUSION}

To conclude this paper, we have provided a detailed field theoretic renormalization analysis of reduced QED up to two loops. The main focus was on reduced $\mathrm{QED}_{4,3}$ (graphene at the IR Lorentz invariant fixed point) which is somehow intermediate between $\mathrm{QED}_{4}$ and $\mathrm{QED}_{3}$ : it is renormalizable similar to $\mathrm{QED}_{4}$ with $\mathrm{UV}$ divergent fermion self-energy but has a finite photon self-energy similar to $\mathrm{QED}_{3}$. Using the BPHZ prescription, we have provided a simple and clear renormalization of the photon and fermion self-energies. We have straightforwardly recovered the results, previously derived via conventional renormalization, for both the interaction correction to the optical conductivity, Eq. (48), and the anomalous dimension of the fermion field, Eq. (60), thereby lifting any possible ambiguity as to their value at the IR fixed point. This constitutes a necessary prerequisite in order to extend our formalism to higher orders and/or to other models such as model II, Eq. (2), that we will consider in our next investigation [112].

\section{ACKNOWLEDGMENTS}

The work of A. V. K. was supported in part by the Russian Foundation for Basic Research (Grant No. 16-02-00790-a).

\section{APPENDIX: MASTER INTEGRALS}

In this appendix, we recall some of the basic master integrals appearing in the main text.

The one-loop (scalar) propagator-type massless integral is defined as

$$
J(D, p, \alpha, \beta)=\int \frac{\left[\mathrm{d}^{D} k\right]}{k^{2 \alpha}(p-k)^{2 \beta}},
$$

where $\left[\mathrm{d}^{D} k\right]=\mathrm{d}^{D} k /(2 \pi)^{D}, p$ is the external momentum, and $\alpha$ and $\beta$ are arbitrary indices. In Eq. (A1), the momentum dependence is easily extracted from dimensional analysis which allows one to write it in the following form:

$$
J(D, p, \alpha, \beta)=\frac{\left(p^{2}\right)^{D / 2-\alpha-\beta}}{(4 \pi)^{D / 2}} G(D, \alpha, \beta)
$$

where $G(D, \alpha, \beta)$ is the (dimensionless) coefficient function of the diagram,

$$
G(D, \alpha, \beta)=\frac{a(\alpha) a(\beta)}{a(\alpha+\beta-D / 2)}, \quad a(\alpha)=\frac{\Gamma(D / 2-\alpha)}{\Gamma(\alpha)} .
$$

The massless two-loop propagator-type diagram is defined as

$$
\begin{aligned}
J\left(D, p, \alpha_{1}, \alpha_{2}, \alpha_{3}, \alpha_{4}, \alpha_{5}\right) \\
\quad=\int \frac{\left[\mathrm{d}^{D} k\right]\left[\mathrm{d}^{D} q\right]}{(k-p)^{2 \alpha_{1}}(q-p)^{2 \alpha_{2}} q^{2 \alpha_{3}} k^{2 \alpha_{4}}(k-q)^{2 \alpha_{5}}},
\end{aligned}
$$

where the $\alpha_{i}(i=1-5)$ are five arbitrary indices. Similar to the one-loop case, the momentum dependence of Eq. (A4) follows from dimensional analysis which allows one to write this diagram in the form:

$$
J\left(D, p,\left\{\alpha_{i}\right\}\right)=\frac{\left(p^{2}\right)^{D-\sum_{i=1}^{5} \alpha_{i}}}{(4 \pi)^{D}} G\left(D,\left\{\alpha_{i}\right\}\right)
$$

where $G\left(D,\left\{\alpha_{i}\right\}\right)$ is the (dimensionless) coefficient function of the diagram. The expression of $G(D, 1,1,1,1, \alpha)$ for arbitrary $\alpha$ can be found in Ref. [75] and that of $G(D, \alpha, 1, \beta, 1,1)$ for arbitrary $\alpha$ and $\beta$ can be found in Ref. [28].
[1] J. González, F. Guinea, and M. A. H. Vozmediano, NonFermi liquid behavior of electrons in the half filled honeycomb lattice (A renormalization group approach), Nucl. Phys. B424, 595 (1994).
[2] G. E. Volovik, The Universe in a Helium Droplet (Oxford University Press, Oxford, UK, 2009).

[3] P. R. Wallace, The band theory of graphite, Phys. Rev. 71, 622 (1947). 
[4] G. W. Semenoff, Condensed Matter Simulation of a Threedimensional Anomaly, Phys. Rev. Lett. 53, 2449 (1984).

[5] J. B. Marston and I. Affleck, Large- $n$ limit of the HubbardHeisenberg model, Phys. Rev. B 39, 11538 (1989).

[6] L. B. Ioffe and A. I. Larkin, Gapless fermions and gauge fields in dielectrics, Phys. Rev. B 39, 8988 (1989).

[7] K. S. Novoselov, A. K. Geim, S. V. Morozov, D. Jiang, M. I. Katsnelson, I. V. Grigorieva, S. V. Dubonos, and A. A. Firsov, Two-dimensional gas of massless Dirac fermions in graphene, Nature (London) 438, 197 (2005).

[8] M. Polini, F. Guinea, M. Lewenstein, H. C. Manoharan, and V. Pellegrini, Artificial honeycomb lattices for electrons, atoms and photons, Nat. Nanotechnol. 8, 625 (2013).

[9] M.Z. Hasan and C. L. Kane, Colloquium: Topological insulators, Rev. Mod. Phys. 82, 3045 (2010).

[10] Z. K. Liu, B. Zhou, Y. Zhang, Z. J. Wang, H. M. Weng, D. Prabhakaran, S.-K. Mo, Z. X. Shen, Z. Fang, X. Dai, Z. Hussain, and Y. L. Chen, Discovery of a three-dimensional topological Dirac semimetal, Na3Bi, Science 343, 864 (2014).

[11] M. Neupane, S.-Y. Xu, R. Sankar, N. Alidoust, G. Bian, C. Liu, I. Belopolski, T.-R. Chang, H.-T. Jeng, H. Lin, A. Bansil, F. Chou, and M. Z. Hasan, Observation of a threedimensional topological Dirac semimetal phase in highmobility $\mathrm{Cd}_{3} \mathrm{As}_{2}$, Nat. Commun. 5, 3786 (2014).

[12] Z. K. Liu, J. Jiang, B. Zhou, Z. J. Wang, Y. Zhang, H. M. Weng, D. Prabhakaran, S.-K. Mo, H. Peng, P. Dudin, T. Kim, M. Hoesch, Z. Fang, X. Dai, Z. X. Shen, D. L. Feng, Z. Hussain, and Y.L. Chen, A stable three-dimensional topological Dirac semimetal $\mathrm{Cd}_{3} \mathrm{As}_{2}$, Nat. Mater. 13, 677 (2014).

[13] S. Borisenko, Q. Gibson, D. Evtushinsky, V. Zabolotnyy, B. Büchner, and R. J. Cava, Experimental Realization of a Three-Dimensional Dirac Semimetal, Phys. Rev. Lett. 113, 027603 (2014).

[14] S.-M. Huang, S.-Y. Xu, I. Belopolski, C.-C. Lee, G. Chang, B. Wang, N. Alidoust, G. Bian, M. Neupane, C. Zhang, S. Jia, A. Bansil, H. Lin, and M. Z. Hasan, A Weyl Fermion semimetal with surface Fermi arcs in the transition metal monopnictide TaAs class, Nat. Commun. 6, 7373 (2015).

[15] S.-Y. Xu et al., Discovery of a Weyl fermion state with Fermi arcs in niobium arsenide, Nat. Phys. 11, 748 (2015).

[16] T. O. Wehling, A. M. Black-Schaffer, and A. V. Balatsky, Dirac materials, Adv. Phys. 63, 1 (2014).

[17] W. Pan, W. Kang, K. W. Baldwin, K. W. West, L. N. Pfeiffer, and D.C. Tsui, Berry phase and anomalous transport of the composite fermions at the half-filled Landau level, Nat. Phys. 13, 1168 (2017).

[18] E. V. Gorbar, V.P. Gusynin, and V. A. Miransky, Dynamical chiral symmetry breaking on a brane in reduced QED, Phys. Rev. D 64, 105028 (2001).

[19] E. C. Marino, Quantum electrodynamics of particles on a plane and the Chern-Simons theory, Nucl. Phys. B408, 551 (1993).

[20] D. T. Son, Is the Composite Fermion a Dirac Particle?, Phys. Rev. X 5, 031027 (2015).

[21] P. Dimopoulos, K. Farakos, C. P. Korthals-Altes, G. Koutsoumbas, and S. Nicolis, Phase structure of the 5-D
Abelian Higgs model with anisotropic couplings, J. High Energy Phys. 02 (2001) 005.

[22] D. B. Kaplan, J. W. Lee, D. T. Son, and M. A. Stephanov, Conformality lost, Phys. Rev. D 80, 125005 (2009).

[23] N. Dorey and N. E. Mavromatos, QED in three-dimension and two-dimensional superconductivity without parity violation, Nucl. Phys. B386, 614 (1992).

[24] A. Kovner and B. Rosenstein, Kosterlitz-Thouless mechanism of two-dimensional superconductivity, Phys. Rev. B 42, 4748 (1990).

[25] S. Teber, Electromagnetic current correlations in reduced quantum electrodynamics, Phys. Rev. D 86, 025005 (2012).

[26] A. V. Kotikov and S. Teber, Note on an application of the method of uniqueness to reduced quantum electrodynamics, Phys. Rev. D 87, 087701 (2013).

[27] I. F. Herbut and V. Mastropietro, Universal conductivity of graphene in the ultrarelativistic regime, Phys. Rev. B 87, 205445 (2013).

[28] A. V. Kotikov and S. Teber, Two-loop fermion self-energy in reduced quantum electrodynamics and application to the ultrarelativistic limit of graphene, Phys. Rev. D 89, 065038 (2014).

[29] S. Teber, Two-loop fermion self-energy and propagator in reduced $\mathrm{QED}_{3,2}$, Phys. Rev. D 89, 067702 (2014).

[30] S. Teber and A. V. Kotikov, Teor. Mat. Fiz. 190, 519 (2017) [The method of uniqueness and the optical conductivity of graphene: New application of a powerful technique for multi-loop calculations, Theor. Math. Phys. 190, 446 (2017)].

[31] D. Valenzuela, S. Hernández-Ortiz, M. Loewe, and A. Raya, Graphene transparency in weak magnetic fields, J. Phys. A 48, 065402 (2015).

[32] S. Hernández-Ortiz, D. Valenzuela, A. Raya, and S. Sánchez-Madrigal, Light absorption in distorted graphene, Int. J. Mod. Phys. 30, 1650084 (2016).

[33] E. C. Marino, L. O. Nascimento, V. S. Alves, and C. M. Smith, Interaction Induced Quantum Valley Hall Effect in Graphene, Phys. Rev. X 5, 011040 (2015).

[34] S. H. Kooi, N. Menezes, V. S. Alves, and C. M. Smith, Quantum valley Hall effect in massive Dirac systems coupled to a scalar field, arXiv:1702.02812.

[35] A. V. Kotikov and S. Teber, Critical behavior of reduced $\mathrm{QED}_{4,3}$ and dynamical fermion gap generation in graphene, Phys. Rev. D 94, 114010 (2016).

[36] V.S. Alves, R. O. C. Junior, E. C. Marino, and L. O. Nascimento, Dynamical mass generation in pseudo quantum electrodynamics with four-fermion interactions, Phys. Rev. D 96, 034005 (2017).

[37] E. C. Marino, L. O. Nascimento, V.S. Alves, and C. Morais Smith, Unitarity of theories containing fractional powers of the d'Alembertian operator, Phys. Rev. D 90 , 105003 (2014).

[38] A. Ahmad, J. J. Cobos-Martínez, Y. Concha-Sánchez, and A. Raya, Landau-Khalatnikov-Fradkin transformations in reduced quantum electrodynamics, Phys. Rev. D 93, 094035 (2016).

[39] W. H. Hsiao and D. T. Son, Duality and universal transport in a mixed-dimension electrodynamics, Phys. Rev. B 96, 075127 (2017). 
[40] C. P. Herzog and K. W. Huang, Boundary conformal field theory and a boundary central charge, J. High Energy Phys. 10 (2017) 189.

[41] I. F. Herbut, V. Juričić, and O. Vafek, Coulomb Interaction, Ripples, and the Minimal Conductivity of Graphene, Phys. Rev. Lett. 100, 046403 (2008).

[42] E. G. Mishchenko, Minimal conductivity in graphene: Interaction corrections and ultraviolet anomaly, Europhys. Lett. 83, 17005 (2008).

[43] V. Juricic, O. Vafek, and I. F. Herbut, Conductivity of interacting massless Dirac particles in graphene: Collisionless regime, Phys. Rev. B 82, 235402 (2010).

[44] D. E. Sheehy and J. Schmalian, Optical transparency of graphene as determined by the fine-structure constant, Phys. Rev. B 80, 193411 (2009).

[45] S. H. Abedinpour, G. Vignale, A. Principi, M. Polini, W.-K. Tse, and A. H. MacDonald, Drude weight, plasmon dispersion, and ac conductivity in doped graphene sheets, Phys. Rev. B 84, 045429 (2011).

[46] I. Sodemann and M. M. Fogler, Interaction corrections to the polarization function of graphene, Phys. Rev. B 86, 115408 (2012).

[47] G. Gazzola, A. L. Cherchiglia, L. A. Cabral, M. C. Nemes, and M. Sampaio, Conductivity of Coulomb interacting massless Dirac particles in graphene: Regularizationdependent parameters and symmetry constraints, Europhys. Lett. 104, 27002 (2013).

[48] B. Rosenstein, M. Lewkowicz, and T. Maniv, Chiral Anomaly and Strength of the Electron-Electron Interaction in Graphene, Phys. Rev. Lett. 110, 066602 (2013).

[49] S. Teber and A. V. Kotikov, Interaction corrections to the minimal conductivity of graphene via dimensional regularization, Europhys. Lett. 107, 57001 (2014).

[50] J. M. Link, P. P. Orth, D. E. Sheehy, and J. Schmalian, Universal collisionless transport of graphene, Phys. Rev. B 93, 235447 (2016).

[51] D. L. Boyda, V. V. Braguta, M. I. Katsnelson, and M. V. Ulybyshev, Many-body effects on graphene conductivity: Quantum Monte Carlo calculations, Phys. Rev. B 94, 085421 (2016).

[52] T. Stauber, P. Parida, M. Trushin, M. V. Ulybyshev, D. L. Boyda, and J. Schliemann, Interacting Electrons in Graphene: Fermi Velocity Renormalization and Optical Response, Phys. Rev. Lett. 118, 266801 (2017).

[53] D. V. Khveshchenko, Ghost Excitonic Insulator Transition in Layered Graphite, Phys. Rev. Lett. 87, 246802 (2001).

[54] E. V. Gorbar, V. P. Gusynin, V. A. Miransky, and I. A. Shovkovy, Magnetic field driven metal insulator phase transition in planar systems, Phys. Rev. B 66, 045108 (2002).

[55] H. Leal and D. V. Khveshchenko, Excitonic instability in two-dimensional degenerate semimetals, Nucl. Phys. B687, 323 (2004).

[56] D. T. Son, Quantum critical point in graphene approached in the limit of infinitely strong Coulomb interaction, Phys. Rev. B 75, 235423 (2007).

[57] O. Vafek and M. J. Case, Renormalization group approach to two-dimensional Coulomb interacting Dirac fermions with random gauge potential, Phys. Rev. B 77, 033410 (2008).
[58] D. V. Khveshchenko, Massive Dirac fermions in singlelayer graphene, J. Phys. Condens. Matter 21, 075303 (2009).

[59] G.-Z. Liu, W. Li, and G. Cheng, Interaction and excitonic insulating transition in graphene, Phys. Rev. B 79, 205429 (2009).

[60] O. V. Gamayun, E. V. Gorbar, and V. P. Gusynin, Gap generation and semimetal-insulator phase transition in graphene, Phys. Rev. B 81, 075429 (2010).

[61] J. E. Drut and T. A. Lahde, Is Graphene in Vacuum an Insulator?, Phys. Rev. Lett. 102, 026802 (2009).

[62] J. E. Drut and T. A. Lahde, Lattice field theory simulations of graphene, Phys. Rev. B 79, 165425 (2009).

[63] J.E. Drut and T. A. Lahde, Critical exponents of the semimetal-insulator transition in graphene: A Monte Carlo study, Phys. Rev. B 79, 241405 (2009).

[64] J.-R. Wang and G.-Z. Liu, Eliashberg theory of excitonic insulating transition in graphene, J. Phys. Condens. Matter 23, 155602 (2011).

[65] J.-R. Wang and G.-Z. Liu, Dynamic gap generation in graphene under the long-range Coulomb interaction, J. Phys. Condens. Matter 23, 345601 (2011).

[66] J. González, Electron self-energy effects on chiral symmetry breaking in graphene, Phys. Rev. B 85, 085420 (2012).

[67] J.-R. Wang and G.-Z. Liu, Absence of dynamical gap generation in suspended graphene, New J. Phys. 14, 043036 (2012).

[68] P. V. Buividovich and M. I. Polikarpov, Monte Carlo study of the electron transport properties of monolayer graphene within the tight-binding model, Phys. Rev. B 86, 245117 (2012).

[69] M. V. Ulybyshev, P. V. Buividovich, M. I. Katsnelson, and M. I. Polikarpov, Monte Carlo Study of the SemimetalInsulator Phase Transition in Monolayer Graphene with Realistic Interelectron Interaction Potential, Phys. Rev. Lett. 111, 056801 (2013).

[70] C. Popovici, C. S. Fischer, and L. von Smekal, Fermi velocity renormalization and dynamical gap generation in graphene, Phys. Rev. B 88, 205429 (2013).

[71] J. González, Phase diagram of the quantum electrodynamics of two-dimensional and three-dimensional Dirac semimetals, Phys. Rev. B 92, 125115 (2015).

[72] A. A. Katanin, Effect of vertex corrections on the possibility of chiral symmetry breaking induced by long-range Coulomb repulsion in graphene, Phys. Rev. B 93, 035132 (2016).

[73] M. E. Carrington, C. S. Fischer, L. von Smekal, and M. H. Thoma, The role of frequency dependence in dynamical gap generation in graphene, Acta Phys. Pol. B Proc. Suppl. 10, 519 (2017).

[74] D. I. Kazakov, The method of uniqueness, a new powerful technique for multiloop calculations, Phys. Lett. 133B, 406 (1983).

[75] A. V. Kotikov, The Gegenbauer polynomial technique: The evaluation of a class of Feynman diagrams, Phys. Lett. B 375, 240 (1996).

[76] D. I. Kazakov, Analytical methods for multiloop calculations: Two lectures on the method of uniqueness, Report No. JINR-E2-84-410. 
[77] A. V. Kotikov and S. Teber, New results for a two-loop massless propagator-type Feynman diagram, arXiv:1611 .07240 .

[78] N. N. Bogoliubov and O. S. Parasiuk, On the multiplication of the causal function in the quantum theory of fields, Acta Math. 97, 227 (1957).

[79] K. Hepp, Proof of the Bogolyubov-Parasiuk theorem on renormalization, Commun. Math. Phys. 2, 301 (1966).

[80] W. Zimmermann, Convergence of Bogoliubov's method of renormalization in momentum space, Commun. Math. Phys. 15, 208 (1969); W. Zimmermann, in Lectures on Elementary Particle and Quantum Field Theory, Brandies University Summer Institute in Theoretical Physics (MIT Press, Cambridge, MA, 1970).

[81] J. C. Collins, Renormalization: An Introduction to Renormalization, the Renormalization Group, and the Operator Product Expansion, Cambridge Monographs on Mathematical Physics (Cambridge University Press, Cambridge, UK, 1986).

[82] Let us also note the more recent Hopf algebraic formulation [83] of renormalization (see Ref. [84] for a recent review). Its application to our model is beyond the scope of our present study.

[83] D. Kreimer, On the Hopf algebra structure of perturbative quantum field theories, Adv. Theor. Math. Phys. 2, 303 (1998).

[84] E. Panzer, Renormalization, Hopf algebras, and Mellin transforms, Contemp. Math. 648, 169 (2015).

[85] D. V. Batkovich and M. Kompaniets, Toolbox for multiloop Feynman diagrams calculations using $R^{*}$ operation, J. Phys. Conf. Ser. 608, 012068 (2015).

[86] F. Herzog and B. Ruijl, The $\mathrm{R}^{*}$-operation for Feynman graphs with generic numerators, J. High Energy Phys. 05 (2017) 037.

[87] H. Isobe and N. Nagaosa, Coulomb Interaction Effect in Weyl Fermions with Tilted Energy Dispersion in Two Dimensions, Phys. Rev. Lett. 116, 116803 (2016).

[88] E. Barnes, E. H. Hwang, R. E. Throckmorton, and S. Das Sarma, Effective field theory, three-loop perturbative expansion, and their experimental implications in graphene many-body effects, Phys. Rev. B 89, 235431 (2014).

[89] J. Hofmann, E. Barnes, and S. Das Sarma, Why Does Graphene Behave as a Weakly Interacting System?, Phys. Rev. Lett. 113, 105502 (2014).

[90] The appearance of fractional d'Alembertian in Eq. (4) for $\varepsilon_{e}>0$ implies that the reduced theory is nonlocal. In the terminology of Gracey [91] the action of the reduced gauge field seems to be a "localizable non-locality" because it can be written as a finite number of local operators as in Eq. (3).

[91] J. A. Gracey, Practicalities of renormalizing quantum field theories, arXiv:hep-th/0605037.

[92] Fractional d'Alembertians (or Laplacians) appear in the field of fractional calculus; see Ref. [93] for an extended monograph. In mathematics, there is a trick apparently due to Caffarelli and Silvestre [94] which amounts to rewriting the fractional field theory in $d$-dimensional space as a local theory in a $d+1$-dimensional space; in our frame, this is nothing else but simply going from (4) back to (3) in the peculiar case $\varepsilon_{e}=1 / 2$. See also Ref. [95] for a nice account on the conformal invariance of ("localizable") nonlocal field theories and Refs. [96-98] for recent references on nonlocal QFTs which make explicit use of the Caffarelli-Silvestre trick and applications to cuprates.

[93] S. Samko, A. A. Kilbas, and O. Marichev, Fractional Integrals and Derivatives (Taylor \& Francis, London, 1993).

[94] L. Caffarelli and L. Silvestre, An extension problem related to the fractional Laplacian, Commun. Partial Differ. Equ. 32, 1245 (2007).

[95] M. A. Rajabpour, Conformal symmetry in non-local field theories, J. High Energy Phys. 06 (2011) 076.

[96] K. Limtragool and P. W. Phillips, Anomalous dimension of the electrical current in the normal state of the cuprates from the fractional Aharonov-Bohm effect, arXiv:1601 .02340 .

[97] G. La Nave and P. Phillips, Exact form of boundary operators dual to interacting bulk scalar fields in the AdS/CFT correspondence, arXiv:1702.00038.

[98] G. La Nave and P. Phillips, Anomalous dimensions for boundary conserved currents in holography via the Caffarelli-Silvestri mechanism for $p$-forms, arXiv:1708.00863.

[99] Such an approach is quite general and may also be applied to phenomena with external fields not mentioned in the Introduction such as, e.g., magnetic catalysis; see Ref. [100,101] for reviews and, e.g., Refs. [102,103] for recent works related to model II.

[100] V. A. Miransky and I. A. Shovkovy, Quantum field theory in a magnetic field: From quantum chromodynamics to graphene and Dirac semimetals, Phys. Rep. 576, 1 (2015).

[101] V. P. Gusynin, Graphene and quantum electrodynamics, Probab. Atomic Sci. Technol. 2013N3, 29 (2013).

[102] C. DeTar, C. Winterowd, and S. Zafeiropoulos, Magnetic Catalysis in Graphene Effective Field Theory, Phys. Rev. Lett. 117, 266802 (2016).

[103] C. DeTar, C. Winterowd, and S. Zafeiropoulos, Lattice field theory study of magnetic catalysis in graphene, Phys. Rev. B 95, 165442 (2017).

[104] D. V. Shirkov, Nonlocal renormalization "stopping" the running gauge, Nucl. Phys. B332, 425 (1990).

[105] It seems therefore that reduced QED provides a simple and concrete example of the nonlocal field theories studied in the recent Refs. [97,98] and invoked phenomenologically in Ref. [96] without knowing the corresponding higher dimensional model.

[106] S. Weinberg, High-energy behavior in quantum field theory, Phys. Rev. 118, 838 (1960).

[107] A. N. Vasil'ev, The Field Theoretic Renormalization Group in Critical Behavior Theory and Stochastic Dynamics (Chapman and Hall/CRC, London, 2004).

[108] C. Di Castro and W. Metzner, Ward Identities and the $\beta$ Function in the Luttinger Liquid, Phys. Rev. Lett. 67, 3852 (1991).

[109] W. Metzner and C. Di Castro, Conservation laws and correlation functions in the Luttinger liquid, Phys. Rev. B 47, 16107 (1993).

[110] C. Itzykson and J.B. Zuber, Quantum Field Theory, Dover Books on Physics (Dover Publications, New York, 2012). 
[111] This is the essential difference with respect to the nonrelativistic case where the contributions of subdivergent graphs only partially cancel each other due to nonstandard Ward identities, and their subtraction therefore affects the final result; see Ref. [112].

[112] S. Teber and A. V. Kotikov, Field theoretic renormalization study of interaction corrections to the universal ac conductivity of graphene, arXiv:1802.09898.

[113] A coefficient similar to $\mathcal{C}^{*}$ actually appeared previously in a different context: the work of Gusynin et al. [114] (and, although not explicitly, in even earlier works of Gracey [115] and Kotikov [116]) on 1/ $N$ expansion of $\mathrm{QED}_{3}$. This is due to a mapping relating large- $N \mathrm{QED}_{3}$ to $\mathrm{QED}_{4,3}$ [35].

[114] V. Gusynin, A. Hams, and M. Reenders, Nonperturbative infrared dynamics of three-dimensional QED with four fermion interaction, Phys. Rev. D 63, 045025 (2001).
[115] J. A. Gracey, Electron mass anomalous dimension at $\mathrm{O}\left(1 / N_{f}^{2}\right)$ in quantum electrodynamics, Phys. Lett. B 317, 415 (1993); Computation of critical exponent $\eta$ at $\mathrm{O}\left(1 / N_{f}^{2}\right)$ in quantum electrodynamics in arbitrary dimensions, Nucl. Phys. B414, 614 (1994).

[116] A. V. Kotikov, Zh. Eksp. Teor. Fiz. 58, 785 (1993) [Critical behavior of 3D electrodynamics, Sov. Phys. JETP 58, 731 (1993)]; On the critical behavior of $(2+1)$-dimensional QED, Phys. At. Nucl. 75, 890 (2012).

[117] H. Kissler, Hopf-algebraic renormalization of QED in the linear covariant gauge, Ann. Phys. (Amsterdam) 372, 159 (2016).

[118] We note that using the mapping found in Ref. [35] between large- $N \mathrm{QED}_{3}$ and $\mathrm{QED}_{4,3}$, it is straightforward to show that Eq. (60) is in perfect agreement with the NLO result of Gracey for $\mathrm{QED}_{3}$, Ref. [115]. 